\title{
Metastatic signaling of hypoxia-related genes across TCGA Pan-Cancer types
}

Andrés López-Cortés ${ }^{1,2, \dagger, *}$, Patricia Guevara-Ramírez ${ }^{1, \dagger}$, Santiago Guerrero ${ }^{1}$, Esteban OrtizPrado $^{3}$, Jennyfer M. García-Cárdenas ${ }^{1}$, Ana Karina Zambrano ${ }^{1}$, Isaac Armendáriz-Castillo ${ }^{1}$, Andy Pérez-Villa ${ }^{1}$, Verónica Yumiceba ${ }^{1}$, Nelson Varela ${ }^{2,4}$, Daniel Córdova-Bastidas ${ }^{1}$, Paola E. Leone $^{1}$, César Paz-y-Miño ${ }^{1, *}$

1 Centro de Investigación Genética y Genómica, Facultad de Ciencias de la Salud Eugenio Espejo, Universidad UTE, Mariscal Sucre Avenue, 170129 Quito, Ecuador

2 Red Latinoamericana de Implementación y Validación de Guías Clínicas Farmacogenómicas (RELIVAF-CYTED)

3 OneHealth Research Group, Facultad de Ciencias de la Salud, Universidad de Las Américas, Avenue de los Granados, 170125 Quito, Ecuador

4 Laboratory of Chemical Carcinogenesis and Pharmacogentics, Department of BasicClinical Oncology, Faculty of Medicine, University of Chile, 70111 Santiago, Chile

\section{$\dagger \quad$ These authors contributed equally to the study}

\section{* Correspondence to:}

Andrés López-Cortés

Centro de Investigación Genética y Genómica, Facultad de Ciencias de la Salud Eugenio Espejo, Universidad UTE, Mariscal Sucre Avenue, 170129 Quito, Ecuador.

E-mail: aalc84@gmail.com

César Paz-y-Miño

Centro de Investigación Genética y Genómica, Facultad de Ciencias de la Salud Eugenio Espejo, Universidad UTE, Mariscal Sucre Avenue, 170129 Quito, Ecuador.

E-mail: cesar.pazymino@ute.edu.ec 


\begin{abstract}
Many primary-tumor subregions have low levels of molecular oxygen, termed hypoxia. Hypoxic tumors are at elevated risk for local failure and distant metastasis. Metastatic disease is the leading cause of cancer-related deaths and involves critical interactions between tumor cells and the microenvironment. Here we focused on elucidating the molecular hallmarks of tumor hypoxia that remains poorly defined. To fill this gap, we analyzed the genomic alterations and hypoxia score of 233 hypoxia-related genes of 6,343 individuals across 17 TCGA Pan-Cancer types. In addition, we analyzed a protein-protein interactome (PPi) network and the shortest paths from hypoxic proteins to metastasis. As results, mRNA high alteration was prevalent in all cancer types. Genomic alterations and hypoxia score presented a highest frequency in tumor stage 4 and positive metastasis status in all cancer types. The most significant signaling pathways were HIF-1, ErbB, PI3K-Akt, FoxO, mTOR, Ras and VEGF. The PPi network revealed a strong association among hypoxic proteins, cancer driver proteins and metastasis driver proteins. The analysis of shortest paths revealed 99 ways to spread metastasis signaling from hypoxic proteins. Additionally, we proposed 62 hypoxic genes strongly associated with metastasis and 27 of them with high amount of genomic alterations. Overall, tumor hypoxia may drive aggressive molecular features across cancer types. Hence, we identified potential biomarkers and therapeutic targets regulated by hypoxia that could be incorporated into strategies aimed at improving novel drug development and treating metastasis.
\end{abstract}




\section{INTRODUCTION}

The Nobel Prize in Physiology or Medicine for 2019 was awarded for the discoveries of how cells sense and adapt to oxygen availability. Human cells undergo fundamental shifts in gene expression when there are changes in the oxygen $\left(\mathrm{O}_{2}\right)$ levels around them ${ }^{1}$. These changes in gene expression alter tissue remodeling, cell metabolism, and even organismal responses such as increases in heart rate and ventilation. In 1990's, the Hypoxia Inducible Factor $(H I F)$ was identified, purified and cloned. HIF is a transcription factor that regulates these oxygendependent responses and consisted of two components: HIF-1 $\alpha$ and $A R N T^{2-4}$. In 1995, the von Hippel-Lindau $(V H L)$ tumor suppressor gene was studied and the first full-length clone of the gene was isolated ${ }^{5-8}$. Consequently, it was demonstrated in 1999 that $V H L$ regulates HIF-1 $\alpha$ post-transcriptional and oxygen-sensitivity degradation ${ }^{9,10}$.

Molecular $\mathrm{O}_{2}$ is a key nutrient required for aerobic metabolism to maintain intracellular bioenergetics and as a substrate in numerous organic and inorganic reactions. Hypoxia occurs in a variety of physiological as well as pathological conditions ${ }^{11}$. Half of all solid tumors are characterized by dynamic gradients of $\mathrm{O}_{2}$ distribution and consumption, that is, the tumor environment presents hypoxia subregions due to changes in tumor metabolism that increases $\mathrm{O}_{2}$ demand or irregular tumor vasculature that decreases $\mathrm{O}_{2}$ supply ${ }^{12-18}$. Tumor adaptation to this imbalance between $\mathrm{O}_{2}$ demand and supply is associated with increased genomic instability and poor clinical prognosis ${ }^{15,19}$, development of tumor stem cell-protective niches ${ }^{20,21}$, resistance to radio- and chemotherapy ${ }^{22,23}$, and increased proclivity for distant metastasis ${ }^{24-26}$.

Metastasis is thought to be the ultimate manifestation of a neoplastic cell's evolution towards becoming autonomous from the host, and remains the main cause of cancer-related death ${ }^{27,28}$. The persistence and lethal relapse of disseminated cancer is driven by stem-like cells that have the ability to regenerate tumors in distant sites ${ }^{29-32}$. In fact, cure of most cancers is probable whenever diagnosis occurs before cells have spread beyond the tissue of origin; otherwise, 
cancer is often referred to as incurable ${ }^{33-35}$. According to Welch and Hurst ${ }^{28}$, the four hallmarks of metastasis are motility and invasion, modulate microenvironment, plasticity and colonization. Regarding mechanisms of HIF-mediated metastasis, hypoxia and activation of HIF signaling influence multiple steps within the metastatic cascade, including invasion and migration, intravasation and extravasation, and establishment of the premetastatic niche, as well as survival and growth at the distant site ${ }^{24,36,37}$.

Although hypoxia is an adverse and targetable prognostic feature in multiple cancer types ${ }^{38,39}$, both the protein-protein interactome (PPi) network and the shortest paths from hypoxia-related genes (HRG) to metastasis had not been described. To fill this gap, we evaluated 233 HRG in 6,343 tumors representing 17 different cancer types. We analyzed genomic alterations and Buffa hypoxia scores per tumor stage, metastatic status and ethnicity across multiple tumor types. We analyzed functional enrichment analysis of hypoxic genes. Lastly, we detailed all information about clinical trials.

\section{RESULTS}

\section{OncoPrint of genomic alterations}

We analyzed genomic alterations of 6,343 individuals with 17 different cancer types taken from the Pan-Cancer Atlas (PCA) project from The Cancer Genome Atlas (TCGA) ${ }^{40-49}$. Figure 1A shows the OncoPrint of genomic alterations (mRNA high, mRNA low, copy number variant (CNV) deep deletion, $\mathrm{CNV}$ amplification, fusion gene, inframe mutation, truncating mutation and missense mutation) of the 233 hypoxia-related genes using cBioPortal ${ }^{50,51}$. The TCGA PanCancer types were bladder urothelial carcinoma (BLCA) with 369 individuals, breast invasive carcinoma (BRCA) with 991 individuals, cervical squamous cell carcinoma and endocervical carcinoma (CESC) with 217 individuals, colorectal adenocarcinoma (CRC) with 521 
individuals, esophageal carcinoma (ESCA) with 163 individuals, head and neck squamous cell carcinoma (HNSC) with 431 individuals, kidney renal clear cell carcinoma (KIRC) with 352 individuals, liver hepatocellular carcinoma (LIHC) with 345 individuals, lung adenocarcinoma (LUAD) with 501 individuals, lung squamous cell carcinoma (LUSC) with 464 individuals, mesothelioma (MESO) with 80 individuals, pancreatic adenocarcinoma (PAAD) with 166 individuals, prostate adenocarcinoma (PRAD) with 481 individuals, skin cutaneous melanoma (SKCM) with 258 individuals, stomach adenocarcinoma (STAD) with 399 individuals, testicular germ cell tumors (TGCT) with 127 individuals and thyroid carcinoma (THCA) with 478 individuals.

We found 100,643 genomic alterations in 6,343 individuals. The genomic alteration with the highest frequency mean was mRNA high (0.047), followed by CNV amplification (0.010), mRNA low (0.004), putative driver mutation (0.003), CNV deep deletion (0.003) and fusion gene (0.0004) (Figure 1B and Table S1).

Consequently, genes with the highest frequency mean per genomic alteration were COPS5 (0.204), ELOC (0.175), ARNT (0.137), PIK3CA (0.135) and TFRC (0.117) with mRNA high; SMAD4 (0.081), MAPK1 (0.044), TP53 (0.034), ELF2 (0.031) and AKT1 (0.031) with mRNA low; PIK3CA (0.064), NDRG1 (0.059), TFRC (0.054), ANGPT1 (0.053) and ARNT (0.049) with CNV amplification; STC1 (0.035), ANGPT2 (0.034), SMAD4 (0.021), TEK (0.016) and VEGFC (0.015) with CNV deep deletion; TP53 (0.391), PIK3CA (0.134), SMAD4 (0.029), VHL (0.026) and $C D H 1$ (0.023) with putative driver mutations; ERBB2 (0.006), PRKCA (0.004), TP53 (0.002), CREBBP (0.002) and STAT3 (0.002) with fusion genes; finally, TP53 (0.476), PIK3CA (0.341), COPS5 (0.246), ELOC (0.213) and ARNT (0.198) with all genomic alterations (Figure 1C and Table S1).

\section{Genomic alterations per TCGA Pan-Cancer type}


Figure 2A shows the frequency mean of genomic alterations per TCGA Pan-Cancer type (0.067). The top ten TCGA Pan-Cancer types with the highest frequency mean of genomic alterations were ESCA (0.089), BRCA (0.080), LUSC (0.080), BLCA (0.077), LUAD (0.076), STAD (0.072), LIHC (0.071), CESC (0.069), HNSC (0.067) and CRC (0.066) (Tables S2-S18).

TCGA Pan-Cancer types with the highest frequency mean per genomic alteration were ESCA (0.056), LUSC (0.053) and MESO (0.052) with mRNA high; PRAD (0.006), CRC (0.006) and LIHC (0.005) with mRNA low; ESCA (0.019), BRCA (0.018) and BLCA (0.016) with CNV amplification; KIRC (0.007), ESCA (0.006) and BLCA (0.005) with CNV deep deletion; CRC (0.005), ESCA (0.005) and MESO (0.005) with putative driver mutations; and BRCA (0.0007), LUAD (0.0006) and KIRC (0.0005) with fusion genes (Figure 2B).

\section{Tumor stage and metastasis status}

Figure 3A shows the frequency mean of genomic alterations of 17 TCGA Pan-Cancer types per tumor stage. The frequency mean of T1 stage was $0.060(n=1,396)$, of T2 stage was $0.072(n=$ 2,306), of T3 stage was $0.068(n=2,029)$, and of T4 was $0.072(n=612)$ (Table S19-S22). The Mann-Whitney $U$ test showed a significant difference of genomic alterations between $\mathrm{T} 1$ and T4 stages $(\mathrm{P}<0.001)$.

Figure 3B shows the association between genomic alterations and the tumor stage with the highest frequency mean. T2 presented the highest frequency mean of CNV amplifications (0.012), CNV deep deletions (0.004) and fusion genes (0.0004); T3 presented the highest frequency mean of mRNA low; and T4 presented the highest frequency mean of mRNA high (0.050) and putative driver mutations (0.004).

On the other hand, figure $3 \mathrm{C}$ shows the frequency mean of genomic alterations per metastasis status. The frequency mean of M0 was $0.069(n=4,254)$ and of M1 was $0.072(n=263)$ (Table 
S23). The Mann-Whitney $U$ test showed a significant difference of genomic alterations between M0 and M1 ( $<<0.001)$.

\section{Hypoxia score}

We first quantified tumor hypoxia in 5,249 tumors from 13 tumor types in the PCA and TCGA

by using Buffa signature ${ }^{52}$. Figure 4A shows the mean of Buffa hypoxia score (HS) per cancer type. The most hypoxic TCGA Pan-Cancer types were HNSC (29.6), LUSC (26.8), CESC (21), CRC (17.3), BLCA (14.5), SKCM (5), KIRC (2.2), LUAD (-0.5), PAAD (-8.5) and BRCA (9.6). The overall mean of HS in the 13 TCGA Pan-Cancer types was 0.85 .

Figure 4B shows box plots related to the Buffa HS mean per tumor stage (T1-T4) in 5,249 individuals with 13 different TCGA Pan-Cancer types. The HS mean in T1 was -4.6, in T2 was 1.2, in $\mathrm{T} 3$ was 0.7 , and in $\mathrm{T} 4$ was 14.5 . Consequently, we obtained a significant difference of hypoxia scores between $\mathrm{T} 1$ and $\mathrm{T} 4$ using the Mann-Whitney $U$ test $(\mathrm{P}<0.001)$.

Figure 4C shows box plots of Buffa HS in 3,871 individuals with metastasis status (M0 and M1). The HS mean in individuals without metastasis (M0) was 2.2, while in individuals with metastasis (M1) was 8.3. Consequently, we obtained a significant difference between the hypoxia scores of M0 and M1 (Mann-Whitney $U$ test, $\mathrm{P}<0.001$ ).

Finally, figure 4D shows box plots of Buffa HS in 3,508 individuals with both ethnicity information and metastasis status. The HS mean in black / African Americans was 4.4, in white individuals was 2.2, and in Asians was -3.9. Regarding metastasis status per ethnic group, black / African Americans had a HS mean of 3.6 in M0 and an increased 12.3 in M1, white individuals had 1.8 in M0 and an increased 8.1 in M1, and Asians had -3.8 in M0 and a decreased -12.3 in M1. 


\section{Protein expression analysis}

The Human Protein Atlas (HPA) presented a map of the human tissue proteome based on tissue microarray-based immunohistochemistry. HPA has analyzed 175 of 233 hypoxia-related proteins across 15 cancer types, classifying the protein expression in high, medium, low and non-detected ${ }^{53-55}$. As results, AKT3, CAMK2A, PDHA1, PDHB, POLR2A, SMAD4 and SOD3 were proteins with high and medium expression in normal tissues, and non-detected expression in a consensus of 15 cancer types, acting as tumor suppression proteins. Meanwhile, ADAM8, BBC3, CCL2, CDH1, CLDN3, EDN1, EIF4EBP1, RELA and TNC were proteins with high and medium expression in a consensus of 15 cancer types, and non-detected expression in normal tissues, acting as oncogenes (Figure 5 and Table S24).

\section{Hallmarks of cancer}

Figure 6 shows a circos plot of $23(10 \%)$ HRG that are hallmarks of cancer according to Hanahan and Weinberg ${ }^{56}$. The top 10 genes with the highest number of interactions with the hallmarks of cancer were TP53, AKT1, EGFR, EPAS1, HIF1A, MTOR, NOTCH1, VHL, CREBBP and ERBB2. Suppression of growth was promoted by TP53, AKT1, EPAS1, VHL, NDRG1, SMAD3, PIK3R1, and EP300. Escaping immune response to cancer was promoted by EGFR and HIF1A. Cell replicative immortality was promoted by TP53 and NOTCH1. Tumor promoting inflammation was promoted by EPAS1. Angiogenesis was promoted by AKT1, ARNT, EGFR, EPAS1, HIF1A, MTOR, NOTCH1, PIK3CA and PLCG1. Escaping programmed cell death was promoted by TP53, AKT1, EGFR, EPAS1, HIF1A, MTOR, NOTCH1, CREBBP, ERBB2, PIK3CA, PLCG1, SMAD3, MAPK1, ATPIA1 and PIK3CB. Change of cellular energetics was promoted by TP53, AKT1, EGFR, HIF1A, MTOR, NOTCH1, VHL, ERBB2, $A R N T, M A P K 1$ and CDH1. Proliferative signaling was promoted by AKT1, EGFR, EPAS1, MTOR, NOTCH1, CREBBP, ERBB2, NDRG1, PIK3CA, PLCG1, SMAD3 and CXCR4. Lastly, invasion and metastasis was promoted by TP53, AKT1, EGFR, EPAS1, HIF1A, MTOR, VHL, 
CREBBP, ERBB2, NDRG1, PIK3CA, PLCG1, SMAD3, ARNT, MAPK1, PIK3R1, ATP1A1, CDH1 and CXCR4.

\section{Protein-protein interactome network}

The PPi network was performed to better understand the behavior of hypoxia-related proteins in cancer and metastasis using the String Database and Cytoscape (Figure 7) ${ }^{57,58}$. With the indicated cutoff of 0.9 , the final interactome network had 285 nodes conformed by 163 (57\%) hypoxia-related proteins, 98 (34\%) cancer driver proteins, and 24 (8\%) hypoxia-related proteins and cancer driver proteins.

The most significant pathways that make up the PPi network were HIF-1 signaling pathway $\left(\mathrm{P}_{\mathrm{adj}}=8.2 \times 10^{-156}\right)$ with 105 nodes, PI3K-Akt signaling pathway $\left(\mathrm{P}_{\mathrm{adj}}=7.5 \times 10^{-50}\right)$ with 85 nodes, FoxO signaling pathway $\left(\mathrm{P}_{\mathrm{adj}}=1.9 \times 10^{-33}\right)$ with 45 nodes, MAPK signaling pathway $\left(\mathrm{P}_{\mathrm{adj}}=3.7 \times 10^{-30}\right)$ with 60 nodes, and Ras signaling pathway $\left(\mathrm{P}_{\mathrm{adj}}=3.2 \times 10^{-30}\right)$ with 54 nodes (Table S25). Additionally, top ten proteins with the highest degree centrality in the HIF-1 signaling pathway were AKT1, PIK3CA, HIF1A, VEGFA, EGFR, EP300, CREBBP, STAT3, MAPK1 and INS; in the PI3K-Akt signaling pathway were TP53, AKT1, PIK3CA, VEGFA, EGFR, RAC1, HRAS, CCND1, MAPK1 and INS; in the FoxO signaling pathway were AKT1, EGFR, HRAS, EP300, CREBBP, STAT3, CCND1, MAPK1, INS and TGFB1; in MAPK signaling pathway were TP53, AKT1, EGFR, RAC1, HRAS, MAPK1, INS, TGFB1, NFKB1 and MAPK3; in the Ras signaling pathway were AKT1, PIK3CA, VEGFA, EGFR, RAC1, HRAS, MAPK1, PTPN11, INS and PLCG1. Lastly, the top ten proteins with the highest degree centrality in the entire PPi network were TP53, AKT1, PIK3CA, HIF1A, VEGFA, EGFR, RAC1, HRAS, EP300 and CREBBP.

On the other hand, the Human Cancer Metastasis Database (HCMDB) is an integrated database designed to analyze large scale expression data of cancer metastasis ${ }^{59}$. Our PPi network was 
conformed by 179 (62\%) metastatic driver proteins (Figure 7) (Table S26). The top tenmetastasis driver proteins with the highest centrality degree were TP53, AKT1, PIK3CA, HIF1A, VEGFA, EGFR, RAC1, HRAS, EP300 and STAT3.

\section{Functional enrichment analysis using g:Profiler}

$\mathrm{g}$ :Profiler searches for a collection of proteins representing pathways, networks, gene ontology

(GO) terms and disease phenotypes such as cancer ${ }^{60,61}$. Figure $8 \mathrm{~A}$ shows the enrichment map of the 233 hypoxia-related proteins. The most significant GO: molecular functions with Benjamini-Hochberg correction and false discovery rate (FDR) $<0.001$ were phosphotransferase activity, kinase activity, enzyme binding, signaling receptor binding and small molecule binding. The most significant GO: biological processes were response to hypoxia, response to decreased oxygen levels, response to oxygen levels, response to abiotic stimulus and response to stress. Lastly, the most significant signaling pathways from the Kyoto Encyclopedia of Genes and Genomes (KEGG) were HIF-1 signaling pathway, ErbB signaling pathway, PI3K-Akt signaling pathway, FoxO signaling pathway and mTOR signaling pathway (Table S27).

On the other hand, figure $8 \mathrm{~B}$ shows the enrichment map of 285 proteins conformed by the interaction between hypoxia-related proteins and cancer driver proteins. Phosphotransferase activity, kinase activity, enzyme binding, signaling receptor binding and small molecule binding were the most significant GO: molecular functions. Cellular response to chemical stimulus, response to oxygen levels, response to decreased oxygen levels, response to hypoxia and response to abiotic stimulus were the most significant GO: biological processes. Finally, HIF-1 signaling pathway, pathways in cancer, PI3K-Akt signaling pathway, ErbB signaling pathway and FoxO signaling pathway were the most significant KEGG pathways (Table S28).

\section{Shortest paths from hypoxia-related proteins to metastasis}


We analyzed the 233 hypoxia-related proteins by using CancerGeneNet software in order to find the shortest paths to metastasis according to Iannuccelli et al ${ }^{62}$. We found that $99(42 \%)$ proteins had paths to metastasis, of which, 49 proteins had positive regulation with an average distance of 3.0 and an average path length of 4.0, 16 proteins had negative regulation with an average distance of 3.5 and an average path length of 4.6, and 34 proteins with unknown regulation status had an average distance of 3.2 and an average path length of 4.3. The top ten hypoxia-related proteins with the shortest distance to metastasis were BACH1 (0.8), AKT2 (1.6), AKT1 (1.6), CAMK2B (1.7), EGFR (1.7), MAPK1 (1.7), MAPK3 (1.7), PRKCA (1.7), PRKCB (1.7) and EGF (1.8). Lastly, the shortest paths to metastasis of the 99 hypoxia-related proteins are fully detailed in figure 9 and table S29.

\section{Hypoxic proteins related to metastasis}

Figure 10 shows a Venn diagram of $62(27 \%)$ hypoxic proteins with the highest affinity to metastasis phenotype (Table S30). Additionally, 27 (12\%) of them presented a frequency mean of genomic alterations more than the average (>0.068) across 17 TCGA Pan-Cancer types (Table S1).

\section{Clinical trials}

Figure 11 shows the current status of clinical trials of drugs focused on metastasis according to the Open Targets Platform ${ }^{63}$. There were 31 drugs that were being analyzed in 161 clinical trials in 13 of 62 genes. Cancer types with clinical trials focused on metastasis were breast cancer, kidney cancer, liver cancer, colorectal cancer, melanoma and prostate cancer (Figure 11A). EGFR presented the highest number of clinical trials in process, recruiting or completed, followed by FLT1, TEK, ERBB2, IGF1R, PIK3CA, PIK3CG, AKT1, AKT2, AKT3, BCL2, NOTCH1 and TGFBR1 (Figure 11B). Most clinical trials were in phase 2, followed by phase 1 
and 4 (Figure 11C). Antibodies and small molecules were the type of drugs focused on metastasis with 86 and 75 clinical trials, respectively (Figure 11D). Inhibition was the most frequent type of action (165), followed by antagonism (5) (Figure 11E). Tyrosine protein kinase EGFR family was the target class with highest number of clinical trials (94), followed by tyrosine protein kinase VEGF family (36), tyrosine protein kinase Tie family (15), enzyme (6), tyrosine protein kinase InsR family (4), AGC protein kinase AKT family (3), Bcl-2 family (1) and TKL protein kinase STKR type 1 subfamily (1) (Figure 11F). Figure 11G diagrams a Sankey plot comparing the number of clinical trials in metastatic drugs analyzed in different cancer types (Table S31 shows the list of clinical trials of drugs focused on metastasis). Lastly, figure S1 and table S32 shows the list of clinical trials in the entire hypoxia-related genes.

\section{DISCUSSION}

Hypoxia induces a series of biological changes that contribute to tumorigenesis and metastasis 24,64 , and are associated with resistance to chemotherapy, radiation therapy, drug therapy and immunotherapy. Therefore, understanding the effect of hypoxia on multi-omics signatures is crucial to improving the outcomes of cancer therapy ${ }^{65}$.

We analyzed the OncoPrint of 100,643 genomic alterations (233 HRG) of 6,343 individuals across 17 TCGA Pan-Cancer types. Cancer types with the highest frequency mean of genomic alterations were ESCA, BRCA, LUSC, BLCA and LUAD. The genomic alteration with the highest frequency mean in all cancer types was mRNA high because hypoxic tumors trigger an over expression of intracellular signals to adapt to the environment ${ }^{12,66,67}$. The hypoxia-related genes with the highest frequency mean of mRNA high alteration were COPS5, ELOC, ARNT, PIK3CA, TFRC, PSEN2, PLCG1, USF1, E2F1 and NDRG1. Lastly, cancer types with the highest frequency mean of mRNA high alteration were ESCA, LUSC, MESO, CESC and BRCA. 
Regarding genomic alterations per tumor stage across 17 TCGA Pan-Cancer types, T4 stage showed the highest frequency mean (0.072), followed by T2, T3 and T1. There was a significant difference (Mann-Whitney $U$ test, $\mathrm{P}<0.001$ ) between $\mathrm{T} 1$ and T4 stages. In addition, T4 stage presented the highest frequency means of mRNA high alteration (0.050) and putative driver mutations (0.004). On the other hand, the frequency mean of genomic alterations in HRG was higher in patients with metastasis (0.072) than in patients without metastasis $(0.069)$, with a significant difference (Mann-Whitney $U$ test, $\mathrm{P}<0.001$ ). That is, the amount of genomic alterations in the HRG increases as the tumor stage and metastasis status evolves.

In order to validate the significant differences of genomic alterations of HRG found in T stages and metastasis status, we analyzed the Buffa hypoxia score across 13 TCGA Pan-Cancer types. The Buffa signature is an approach for deriving signatures that combine knowledge of gene function and analysis of in vivo co-expression patterns. Hypoxia scores were estimated by obtaining the mean expression $(\log 2)$ of 52 hypoxia genes reported by Buffa et al ${ }^{52,68}$. Cancer types with the highest HS mean were HNSC, LUSC, CESC, CRC and BLCA, and the overall HS mean was 0.85 . Regarding T stages, T4 presented the highest HS mean (14.5), followed by T2, T3 and T1. There was a significant difference (Mann-Whitney $U$ test, $\mathrm{P}<0.001$ ) between T1 and T4 stages. On the other hand, the Buffa HS mean in patients with metastasis (8.3) was higher than in patients without metastasis (2.2), with a significant difference (Mann-Whitney $U$ test, $\mathrm{P}<0.001)$. In regard to ethnicity, Guerrero et al., indicate a need to diversify oncological studies to other populations along with novel strategies to enhance race/ethnicity data recording and reporting ${ }^{69}$. In this context, Black / African Americans showed highest HS mean (4.4) than Whites and Asians, and Black /African Americans with metastasis showed highest HS mean (12.3) than Whites (8.1) and Asians (-12.3). Notably, the Buffa HS increases as the tumor stage and metastasis status evolves. 
In addition to analyzing the genomics and transcriptomics of HRG, we evaluated the human tissue proteome based on tissue microarray-based immunohistochemistry of 175 hypoxiarelated proteins across 15 TCGA Pan-Cancer types ${ }^{53-55,67}$. The AKT3, CAMK2A, PDHA1, PDHB, POLR2A, SMAD4 and SOD3 proteins acted as tumor suppressors, meanwhile, the ADAM8, BBC3, CCL2, CDH1, CLDN3, EDN1, EIF4EBP1, RELA and TN proteins acted as oncogenes according to the expression levels between normal and tumor tissues.

Regarding the hallmarks of cancer proposed by Hanahan and Weinberg ${ }^{56}, 23$ of 230 HRG showed some of these cancer features. The TP53, AKT1, EGFR, EPAS1, HIF1A, MTOR, NOTCH1, VHL, CREBBP and ERBB2 genes presented the highest number of associations with these hallmarks. Invasion and metastasis was promoted by TP53, AKT1, EGFR, EPAS1, HIF1A, MTOR, VHL, CREBBP, ERBB2, NDRG1, PIK3CA, PLCG1, SMAD3, ARNT, MAPK1, PIK3R1, ATP1A1, CDH1 and CXCR4; suppressed by TP53,VHL, NDRG1, ARNT and PIK3R1; finally, promoted and suppressed by $A K T 1$ and $C D H 1$.

It is important to mention that proteins do not work alone, that is why we performed a proteinprotein interactome network analysis to better understand the behavior of hypoxia-related proteins and its correlation with cancer driver proteins and metastasis. The PPi network had 285 nodes conformed by 163 (57\%) hypoxic proteins, 98 (34\%) cancer driver proteins, and 24 cancer driver proteins with hypoxic signature. Regarding metastasis, our PPi network was conformed by 179 of $285(62 \%)$ metastatic driver proteins. The top ten-metastasis driver proteins with the highest centrality degree were TP53, AKT1, PIK3CA, HIF1A, VEGFA, EGFR, RAC1, HRAS, EP300 and STAT3.

Consequently, we performed two functional enrichment analyses by using g:Profiler ${ }^{60}$. The first analysis was conformed by the 233 hypoxia-related proteins where the most significant (FDR < 0.001) GO: biological processes were response to hypoxia, response to decreased oxygen levels, response to oxygen levels, response to abiotic stimulus and response to stress. The most 
significant KEGG signaling pathways were HIF-1, ErbB, PI3K-Akt, FoxO and mTOR. As expected, all terms were related to hypoxia conditions. The second analysis showed the enrichment map of 285 proteins conformed by the interaction between hypoxia-related proteins and cancer driver proteins. The most significant GO: biological processes were cellular response to chemical stimulus, response to oxygen levels, response to decreased oxygen levels, response to hypoxia and response to abiotic stimulus. Finally, HIF-1, PI3K-Akt, ErbB and FoxO were the most significant KEGG signaling pathways.

Another way to analyze the correlation between hypoxia and metastasis was unrevealing the shortest paths from hypoxia-related proteins to metastasis signaling ${ }^{62}$. We found that 99 of 233 (42\%) proteins had paths to metastasis where 49 had positive regulation with an average distance of 3.0, and 16 had negative regulation with an average distance of 3.5. Hypoxic proteins with the shortest distance to metastasis were BACH1, AKT2, AKT1, CAMK2B, EGFR, MAPK1, MAPK3, PRKCA, PRKCB and EGF. Lastly, these 99 hypoxia-related proteins send metastatic signaling through CTNNB1, BACH1, EZR, PTTG, WASF3 and NFE2L2.

After the PPi network analysis and the analysis of the shortest paths to metastasis, we proposed 62 proteins with the highest affinity to metastasis phenotype (Figure 10). Of them, 27 proteins presented a frequency mean of genomic alterations more than the average (>0.068) across 17 TCGA Pan-Cancer types. Consequently, we observed that 13 of these 62 proteins have 31 drugs studied in 161 clinical trials focused on metastasis. It is important to mention that the percentage of druggable proteins under clinical trials is low and machine-learning analysis could be required in order to improve the selection of drugs against metastasis ${ }^{70,71}$.

\section{CONCLUSIONS}


Hypoxia and HIF-dependent signaling play an important role in metastasis tumor progression. The hypoxic tumor microenvironment influences both the early and late stages of metastasis. Our study provides a comprehensive view of multi-omics analyses in hypoxia-related genes. Our findings suggest that individuals with metastasis present highest frequency of genomic alterations and hypoxia score across 17 TCGA Pan-Cancer types. The most altered pathways where hypoxic genes are involved are HIF-1, ErbB, PI3K-Akt, FoxO, mTOR and Ras signaling pathways. The analyses of both the PPi network and the shortest paths to metastasis suggest 62 proteins strongly related to metastasis. Finally, there are few drugs anti-metastasis that are being studied in clinical trials so far. Hence, it is imperative to focus our strengths in drug developing and drug discovery to improve precision medicine and find effective therapies anti-metastasis.

\section{METHODS}

\section{Dataset of hypoxia-related genes}

In this study we analyzed a dataset of $233 \mathrm{HRG}$ curated manually. 109 of them conformed the

KEGG HIF-1 signaling pathway ${ }^{72}, 102$ of them were involved in some of the GO terms related to hypoxia: response to hypoxia, cellular response to hypoxia and cellular response to decreased oxygen levels by using g:Profiler ${ }^{60}, 52$ of them were hypoxia genes according to Buffa et al ${ }^{52}$, and 75 of them underlie high-altitude adaptive phenotypes in populations from the Andean Altiplano, Semien Plateau and the Tibetan Plateau, according to Bigham ${ }^{73}$.

\section{Genomic alterations}

Genomic alterations (mRNA high, mRNA low, CNV deep deletion, CNV amplification, fusion gene, inframe mutation, truncating mutation and missense mutation) were analyzed in 6,343 tumors (all with tumor stage) from 17 TCGA Pan-Cancer types (BLCA, BRCA, CESC, CRC, 
ESCA, HNSC, KIRC, LIHC, LUAD, LUSC, MESO, PAAD, PRAD, SKCM, STAD, TGCT and THCA). Genomics and clinical data related to tumor stage (T1-T4) and metastasis status (M0-M1) was taken from the Genomics Data Commons of the National Cancer Institute (https://portal.gdc.cancer.gov/) and the cBioPortal (http://www.cbioportal.org/) ${ }^{50,51}$.

\section{Hypoxia score}

The Buffa hypoxia score was analyzed in 5,249 tumors from 13 TCGA Pan-Cancer types (HNSC, LUSC, CESC, CRC, BLCA, SKCM, KIRC, LUAD, PAAD, BRCA, LIHC, PRAD and THCA) ${ }^{52}$. An approach for deriving signatures that combine knowledge of gene function and analysis of in vivo co-expression patterns was used to define a common hypoxia signature in cancer. Hypoxia scores were estimated by obtaining the mean expression $(\log 2)$ of 52 hypoxia genes reported by Buffa et al ${ }^{52,68}$.

Data related to Buffa hypoxia score, tumor stage, metastasis status and ethnicity (black / African American, white and Asian) was taken from the Genomics Data Commons and the cBioPortal 50,51. Lastly, the Mann-Whitney $U$ test was performed to determine significant differences between hypoxia score and clinical data.

\section{Protein expression analysis}

The Human Protein Atlas (https://www.proteinatlas.org/) explains the diverse molecular signatures of proteomes in the human tissue based on tissue microarray-based immunohistochemistry ${ }^{53,54,74,75}$. We compared the protein levels (high, medium, low, nondetected) of our 175 proteins between normal tissues and tumor tissues from 15 different cancer types. Lastly, we obtained a list of proteins with potential interaction as oncogenes and tumor suppressor genes. 


\section{Protein-protein interactome network}

The PPi network with a highest confidence cutoff of 0.9 and zero node addition was created using the String Database, which takes into account predicted and known interactions ${ }^{57,76,77}$. The degree centrality of a node means the number of edges the node has to other nodes in the network. Metastasis driver proteins that interact with the hypoxia-related proteins were taken from the Human Cancer Metastasis Database (https://hcmdb.i-sanger.com/). HCMDB is an integrated database designed to analyze large scale expression data of cancer metastasis ${ }^{59}$. The network visualization was analyzed through the Cytoscape software ${ }^{58}$. Hypoxia-related proteins, cancer driver proteins, metastasis driver proteins, and significant signaling pathways $(\mathrm{P}<0.001)$ were differentiated by colors in the PPi network.

\section{Functional enrichment analysis using g:Profiler}

The enrichment analysis gives scientists curated interpretation of gene/protein lists from genome-scale experiments ${ }^{60}$. The hypoxia-related proteins and cancer driver proteins were analyzed by using g:Profiler (https://biit.cs.ut.ee/gprofiler/gost) in order to obtain significant annotations (Benjamini-Hochberg $(\mathrm{FDR})<0.001)$ related to GO terms (molecular functions and biological processes), and KEGG signaling pathways ${ }^{60,72}$.

\section{Shortest paths from hypoxia-related proteins to metastasis}

CancerGeneNet (https://signor.uniroma2.it/CancerGeneNet/) is a resource that links genes that are frequently mutated in all cancer types to cancer phenotypes. This resource is based on the annotation of experimental information that permits to embed the cancer genes into the cell network of causal protein relationships curated in SIGNOR ${ }^{78}$. Consequently, this bioinformatics tool allows to infer likely paths of causal interactions linking cancer associated genes to cancer phenotypes such as metastasis ${ }^{62}$. Iannuccelli et al explained that the shortest 
paths between a specific gene and cancer phenotypes was programmatically implemented using the shortest path function of the $\mathrm{R}$ igraph, obtaining a distance score and a path length score ${ }^{62,79}$. Hence, we analyzed the shortest paths from our hypoxia-related proteins to metastasis to better understand the association to this hallmark of cancer ${ }^{62,80}$.

\section{Clinical trials}

The Open Targets Platform (https://www.targetvalidation.org) is comprehensive and robust data integration for access to and visualization of potential drug targets associated with several cancer types and metastasis. Additionally, this platform shows all drugs in clinical trials associated with biomarkers or cancer genes, detailing its phase, type of drug, action type, and target class ${ }^{63}$. 


\section{Acknowledgments}

Universidad UTE (Ecuador) and the Latin American Society of Pharmacogenomics and Personalized Medicine (SOLFAGEM) supported this research.

\section{Author contribution}

ALC and PGR conceived the subject and the conceptualization of the study. ALC wrote the manuscript. CPyM supervised the project. ALC and CPyM did founding acquisition. PGR, SG, JMGC, AKZ, IAC, APV, VY, DCB and PEL did data curation and supplementary data. SG, EOP and NV gave conceptual advice and valuable scientific input. Finally, all authors reviewed and approved the manuscript.

\section{Competing interests}

The authors declare no competing interests.

\section{Data availability statement}

All data generated or analyzed during this study are included in this published article (and its Supplementary Information files). 


\section{REFERENCES}

1. Giaccia, A. J., Simon, M. C. \& Johnson, R. The biology of hypoxia: The role of oxygen sensing in development, normal function, and disease. in Genes and Development (2004). doi:10.1101/gad.1243304

2. Wang, G. L., Jiang, B. H., Rue, E. A. \& Semenza, G. L. Hypoxia-inducible factor 1 is a basic-helix-loop-helix-PAS heterodimer regulated by cellular $\mathrm{O} 2$ tension. Proc. Natl. Acad. Sci. U. S. A. (1995). doi:10.1073/pnas.92.12.5510

3. Wang, G. L. \& Semenza, G. L. General involvement of hypoxia-inducible factor 1 in transcriptional response to hypoxia. Proc. Natl. Acad. Sci. U. S. A. (1993). doi:10.1073/pnas.90.9.4304

4. Wang, G. L. \& Semenza, G. L. Purification and characterization of hypoxia-inducible factor. J. Biol. Chem. (1995). doi:10.1074/jbc.270.3.1230

5. Ohh, M. et al. Ubiquitination of hypoxia-inducible factor requires direct binding to the $\beta$-domain of the von Hippel - Lindau protein. Nat. Cell Biol. (2000). doi:10.1038/35017054

6. Lonergan, K. M. et al. Regulation of Hypoxia-Inducible mRNAs by the von HippelLindau Tumor Suppressor Protein Requires Binding to Complexes Containing Elongins B/C and Cul2. Mol. Cell. Biol. (1998). doi:10.1128/mcb.18.2.732

7. Kibel, A., Iliopoulos, O., DeCaprio, J. A. \& Kaelin, W. G. Binding of the von HippelLindau tumor suppressor protein to Elongin B and C. Science (80-. ). (1995). doi:10.1126/science.7660130

8. Iliopoulos, O., Kibel, A., Gray, S. \& Kaelin, W. G. Tumour suppression by the human von hippel-lindau gene product. Nat. Med. (1995). doi:10.1038/nm0895-822

9. Ivan, M. et al. HIF $\alpha$ targeted for VHL-mediated destruction by proline hydroxylation: Implications for O2 sensing. Science (80-. ). (2001). doi:10.1126/science.1059817

10. Maxwell, P. H. et al. The tumour suppressor protein VHL targets hypoxia-inducible factors for oxygen-dependent proteolysis. Nature (1999). doi:10.1038/20459

11. Xie, H. \& Simon, M. C. Oxygen availability and metabolic reprogramming in cancer. Journal of Biological Chemistry (2017). doi:10.1074/jbc.R117.799973

12. Bhandari, V. et al. Molecular landmarks of tumor hypoxia across cancer types. Nat. Genet. (2019). doi:10.1038/s41588-018-0318-2

13. Wilson, W. R. \& Hay, M. P. Targeting hypoxia in cancer therapy. Nature Reviews Cancer (2011). doi:10.1038/nrc3064

14. Harris, A. L. Hypoxia - A key regulatory factor in tumour growth. Nature Reviews Cancer (2002). doi:10.1038/nrc704

15. Bristow, R. G. \& Hill, R. P. Hypoxia and metabolism: Hypoxia, DNA repair and genetic instability. Nature Reviews Cancer (2008). doi:10.1038/nrc2344

16. Dhani, N., Fyles, A., Hedley, D. \& Milosevic, M. The clinical significance of hypoxia in human cancers. Seminars in Nuclear Medicine (2015). doi:10.1053/j.semnuclmed.2014.11.002

17. Brown, J. M. \& Wilson, W. R. Exploiting tumour hypoxia in cancer treatment. Nature Reviews Cancer (2004). doi:10.1038/nrc1367

18. Mucaj, V., Shay, J. E. S. \& Simon, M. C. Effects of hypoxia and HIFs on cancer metabolism. Int. J. Hematol. (2012). doi:10.1007/s12185-012-1070-5

19. Luoto, K. R., Kumareswaran, R. \& Bristow, R. G. Tumor hypoxia as a driving force in genetic instability. Genome Integrity (2013). doi:10.1186/2041-9414-4-5

20. Mohyeldin, A., Garzón-Muvdi, T. \& Quiñones-Hinojosa, A. Oxygen in stem cell biology: A critical component of the stem cell niche. Cell Stem Cell (2010). doi:10.1016/j.stem.2010.07.007

21. Eliasson, P. \& Jönsson, J. I. The hematopoietic stem cell niche: Low in oxygen but a nice place to be. Journal of Cellular Physiology (2010). doi:10.1002/jcp.21908

22. Brizel, D. M., Dodge, R. K., Clough, R. W. \& Dewhirst, M. W. Oxygenation of head and neck cancer: Changes during radiotherapy and impact on treatment outcome. Radiother. Oncol. (1999). doi:10.1016/S0167-8140(99)00102-4 
23. Nordsmark, M. \& Overgaard, J. Tumor hypoxia is independent of hemoglobin and prognostic for loco-regional tumor control after primary radiotherapy in advanced head and neck cancer. Acta Oncol. (Madr). (2004). doi:10.1080/02841860410026189

24. Rankin, E. B. \& Giaccia, A. J. Hypoxic control of metastasis. Science (2016). doi:10.1126/science.aaf4405

25. Gilkes, D. \& Semenza, G. Role of hypoxia-inducible factors in breast cancer metastasis. Futur. Oncol. 9, 1623-1636 (2015).

26. Zhong, H. et al. Overexpression of hypoxia-inducible factor $1 \alpha$ in common human cancers and their metastases. Cancer Res. (1999).

27. Ganesh, K. et al. L1CAM defines the regenerative origin of metastasis-initiating cells in colorectal cancer. Nat. Cancer 1, (2020).

28. Welch, D. R. \& Hurst, D. R. Defining the Hallmarks of Metastasis. Cancer Research (2019). doi:10.1158/0008-5472.CAN-19-0458

29. Batlle, E. \& Clevers, H. Cancer stem cells revisited. Nature Medicine (2017). doi:10.1038/nm.4409

30. Celià-Terrassa, T. \& Kang, Y. Distinctive properties of metastasis-initiating cells. Genes and Development (2016). doi:10.1101/gad.277681.116

31. Lambert, A. W., Pattabiraman, D. R. \& Weinberg, R. A. Emerging Biological Principles of Metastasis. Cell (2017). doi:10.1016/j.cell.2016.11.037

32. Massagué, J. \& Obenauf, A. C. Metastatic colonization by circulating tumour cells. Nature (2016). doi:10.1038/nature17038

33. Eccles, S. A. \& Welch, D. R. Metastasis: recent discoveries and novel treatment strategies. Lancet (2007). doi:10.1016/S0140-6736(07)60781-8

34. Klein, C. A. Framework models of tumor dormancy from patient-derived observations. Current Opinion in Genetics and Development (2011). doi:10.1016/j.gde.2010.10.011

35. Steeg, P. S. \& Theodorescu, D. Metastasis: A therapeutic target for cancer. Nature Clinical Practice Oncology (2008). doi:10.1038/ncponc1066

36. De Bock, K., Mazzone, M. \& Carmeliet, P. Antiangiogenic therapy, hypoxia, and metastasis: Risky liaisons, or not? Nature Reviews Clinical Oncology (2011). doi:10.1038/nrclinonc.2011.83

37. Semenza, G. L. The hypoxic tumor microenvironment: A driving force for breast cancer progression. Biochimica et Biophysica Acta - Molecular Cell Research (2016). doi:10.1016/j.bbamcr.2015.05.036

38. Janssens, G. O. et al. Accelerated radiotherapy with carbogen and nicotinamide for laryngeal cancer: Results of a phase III randomized trial. J. Clin. Oncol. (2012). doi:10.1200/JCO.2011.35.9315

39. Hoskin, P. J., Rojas, A. M., Bentzen, S. M. \& Saunders, M. I. Radiotherapy with concurrent carbogen and nicotinamide in bladder carcinoma. J. Clin. Oncol. (2010). doi:10.1200/JCO.2010.28.4950

40. Hoadley, K. A. et al. Cell-of-Origin Patterns Dominate the Molecular Classification of 10,000 Tumors from 33 Types of Cancer. Cell 173, 291-304.e6 (2018).

41. Berger, A. C. et al. A Comprehensive Pan-Cancer Molecular Study of Gynecologic and Breast Cancers. Cancer Cell (2018). doi:10.1016/j.ccell.2018.03.014

42. Liu, Y. et al. Comparative Molecular Analysis of Gastrointestinal Adenocarcinomas. Cancer Cell 1-15 (2018). doi:10.1016/j.ccell.2018.03.010

43. Campbell, J. D. et al. Genomic, Pathway Network, and Immunologic Features Distinguishing Squamous Carcinomas. Cell Rep. 194-212 (2018). doi:10.1016/j.celrep.2018.03.063

44. Ricketts, C. J. et al. The Cancer Genome Atlas Comprehensive Molecular Characterization of Renal Cell Carcinoma. Cell Rep. (2018). doi:10.1016/j.celrep.2018.03.075

45. Huang, K. lin et al. Pathogenic Germline Variants in 10,389 Adult Cancers. Cell (2018). doi:10.1016/j.cell.2018.03.039

46. Bailey, M. H. et al. Comprehensive Characterization of Cancer Driver Genes and Mutations. Cell 173, 371-385.e18 (2018). 
47. Gao, Q. et al. Driver Fusions and Their Implications in the Development and Treatment of Human Cancers. Cell Rep. (2018). doi:10.1016/j.celrep.2018.03.050

48. Liu, J. et al. An Integrated TCGA Pan-Cancer Clinical Data Resource to Drive HighQuality Survival Outcome Analytics. Cell (2018). doi:10.1016/j.cell.2018.02.052

49. Sanchez-Vega, F. et al. Oncogenic Signaling Pathways in The Cancer Genome Atlas. Cell (2018). doi:10.1016/j.cell.2018.03.035

50. Cerami, E. et al. The cBio cancer genomics portal: an open platform for exploring multidimensional cancer genomics data. Cancer Discov. (2012). doi:10.1158/21598290.CD-12-0095

51. Gao, J. et al. Integrative analysis of complex cancer genomics and clinical profiles using the cBioPortal. Sci. Signal. (2013). doi:10.1126/scisignal.2004088

52. Buffa, F. M., Harris, A. L., West, C. M. \& Miller, C. J. Large meta-analysis of multiple cancers reveals a common, compact and highly prognostic hypoxia metagene. Br. J. Cancer (2010). doi:10.1038/sj.bjc.6605450

53. Thul, P. J. \& Lindskog, C. The human protein atlas: A spatial map of the human proteome. Protein Sci. (2018). doi:10.1002/pro.3307

54. Uhlen, M. et al. A pathology atlas of the human cancer transcriptome. Science (80-. ). (2017). doi:10.1126/science.aan2507

55. Uhlén, M. et al. Tissue-based map of the human proteome. Science (80-. ). (2015). doi:10.1126/science.1260419

56. Hanahan, D. \& Weinberg, R. A. Hallmarks of cancer: The next generation. Cell 144, 646-674 (2011).

57. Szklarczyk, D. et al. STRING v10: protein-protein interaction networks, integrated over the tree of life. Nucleic Acids Res. 43, D447-52 (2015).

58. Shannon, P. et al. Cytoscape: a software environment for integrated models of biomolecular interaction networks. Genome Res. 13, 2498-504 (2003).

59. Zheng, G. et al. HCMDB: The human cancer metastasis database. Nucleic Acids Res. (2018). doi:10.1093/nar/gkx1008

60. Raudvere, U. et al. g:Profiler: a web server for functional enrichment analysis and conversions of gene lists (2019 update). Nucleic Acids Res. (2019).

doi:10.1093/nar/gkz369

61. García-cárdenas, J. M. et al. Post-transcriptional Regulation of Colorectal Cancer $\square$ : A Focus on RNA-Binding Proteins. 6, 1-18 (2019).

62. Iannuccelli, M. et al. CancerGeneNet: linking driver genes to cancer hallmarks. Nucleic Acids Res. (2019). doi:10.1093/nar/gkz871

63. Carvalho-Silva, D. et al. Open Targets Platform: New developments and updates two years on. Nucleic Acids Res. (2019). doi:10.1093/nar/gky1133

64. Gilkes, D. M. \& Semenza, G. L. Role of hypoxia-inducible factors in breast cancer metastasis. Futur. Oncol. 9, 1623-1636 (2013).

65. Ye, Y. et al. Characterization of hypoxia-associated molecular features to aid hypoxiatargeted therapy. Nat. Metab. (2019). doi:10.1038/s42255-019-0045-8

66. Guerrero, S. et al. In silico analyses reveal new putative Breast Cancer RNA-binding proteins. bioRxiv (2020). doi:10.1101/2020.01.08.898965

67. López-Cortés, A. et al. OncoOmics approaches to reveal essential genes in breast cancer: a panoramic view from pathogenesis to precision medicine. bioRxiv (2019). doi:10.1101/638866

68. Chang, W. H., Forde, D. \& Lai, A. G. A novel signature derived from immunoregulatory and hypoxia genes predicts prognosis in liver and five other cancers. J. Transl. Med. (2019). doi:10.1186/s12967-019-1775-9

69. Guerrero, S. et al. Analysis of Racial / Ethnic Representation in Select Basic and Applied Cancer Research Studies. 1-8 (2018). doi:10.1038/s41598-018-32264-x

70. Lopez-Cortes, A. et al. Prediction of breast cancer proteins using molecular descriptors and artificial neural networks: a focus on cancer immunotherapy proteins, metastasis driver proteins, and RNA-binding proteins. bioRxiv Bioinforma. (2019).

doi:10.1101/840108 
71. López-Cortés, A. et al. Prediction of druggable proteins using machine learning and functional enrichment analysis: a focus on cancer-related proteins and RNA-binding proteins. bioRxiv (2019). doi:10.1101/825513

72. Ogata, H. et al. KEGG: Kyoto encyclopedia of genes and genomes. Nucleic Acids Research 27, 29-34 (1999).

73. Bigham, A. W. Genetics of human origin and evolution: high-altitude adaptations. Current Opinion in Genetics and Development (2016). doi:10.1016/j.gde.2016.06.018

74. Uhlen, M. et al. Towards a knowledge-based Human Protein Atlas. Nat. Biotechnol. (2010). doi:10.1038/nbt1210-1248

75. Uhlén, M. et al. A Human Protein Atlas for Normal and Cancer Tissues Based on Antibody Proteomics. Mol. Cell. Proteomics (2005). doi:10.1074/mcp.M500279MCP200

76. López-Cortés, A. et al. Gene prioritization, communality analysis, networking and metabolic integrated pathway to better understand breast cancer pathogenesis. Sci. Rep. 8, 16679 (2018).

77. López-Cortés, A. et al. Pharmacogenomics, biomarker network, and allele frequencies in colorectal cancer. Pharmacogenomics J. (2019). doi:10.1038/s41397-019-0102-4

78. Perfetto, L. et al. SIGNOR: A database of causal relationships between biological entities. Nucleic Acids Res. (2016). doi:10.1093/nar/gkv1048

79. Csardi, G. \& Nepusz, T. The igraph software package for complex network research. InterJournal Complex Syst. (2006).

80. Hanahan, D. \& Weinberg, R. A. Hallmarks of Cancer: The Next Generation. Cell 144, 646-674 (2011). 


\section{Figure legends}

Figure 1. Panoramic landscape of genomic alterations across TCGA Pan-Cancer types. A) OncoPrint of genomic alterations (mRNA high, mRNA low, CNV amplification, CNV deep deletion, putative driver mutation and fusion genes) of the most altered hypoxia-related genes. B) Most frequency genomic alterations across all cancer types. C) Most altered genes per genomic alteration type.

Figure 2. Genomic alterations per TCGA Pan-Cancer type. A) Frequency mean of genomic alterations per cancer type. B) Most altered cancer types per genomic alteration.

Figure 3. Tumor stages and metastasis status. A) Frequency mean of all genomic alterations per tumor stage across 17 Pan-Cancer types. B) Frequency mean of each genomic alteration per tumor stage. C) Frequency mean of all genomic alterations per metastasis status.

Figure 4. Hypoxia score. A) Hypoxia score mean across 13 Pan-Cancer types. B) Hypoxia score mean per tumor stage. C) Hypoxia score mean per metastasis status. D) Hypoxia score mean per metastasis status related to ethnicity.

Figure 5. Molecular signatures of proteomes in the human tissue based on tissue microarraybased immunohistochemistry according to the Human Protein Atlas.

Figure 6. Hypoxia-related genes with hallmark of cancer signatures.

Figure 7. Protein-protein interactome network conformed by hypoxia-related proteins, cancer driver proteins and metastatic driver proteins. 
Figure 8. Functional enrichment analysis using g:Profiler. A) Most significant GO molecular functions, GO biological processes and KEGG signaling pathways of the 233 hypoxia-related genes. B) Most significant GO molecular functions, GO biological processes and KEGG signaling pathways of the 285 hypoxic genes and cancer driver genes.

Figure 9. Metastasis signaling. Shortest paths from 99 hypoxia-related proteins to metastasis.

Figure 10. Venn diagram conformed by the hypoxic proteins with shortest paths to metastasis, and the metastatic proteins from the PPi network.

Figure 11. Clinical trials of drugs focused on metastasis. A) Percentage of clinical trials per cancer type. B) Hypoxic genes with highest number of clinical trials on metastasis. C) Phase of clinical trials. D) Type of drugs. E) Action type of drugs. F) Target class. G) Correlation of clinical trials, cancer types and drugs using a Sankey plot. 


\begin{tabular}{|c|c|c|c|c|c|c|c|c|c|c|c|}
\hline \multirow{2}{*}{$\begin{array}{l}\text { TCGA } \\
\text { BLCA }\end{array}$} & TCGA & TCGA & TCGA & TCGA & TCGA & TCGA & TCGA & TCGA & TCGA & TCG & TCG \\
\hline & $\begin{array}{c}\text { BRCA } \\
n=991\end{array}$ & CESC & CRC & ESCA & HNSC & KIRC & LIHC & LUAD & LUSC & MES & PAA \\
\hline
\end{tabular}

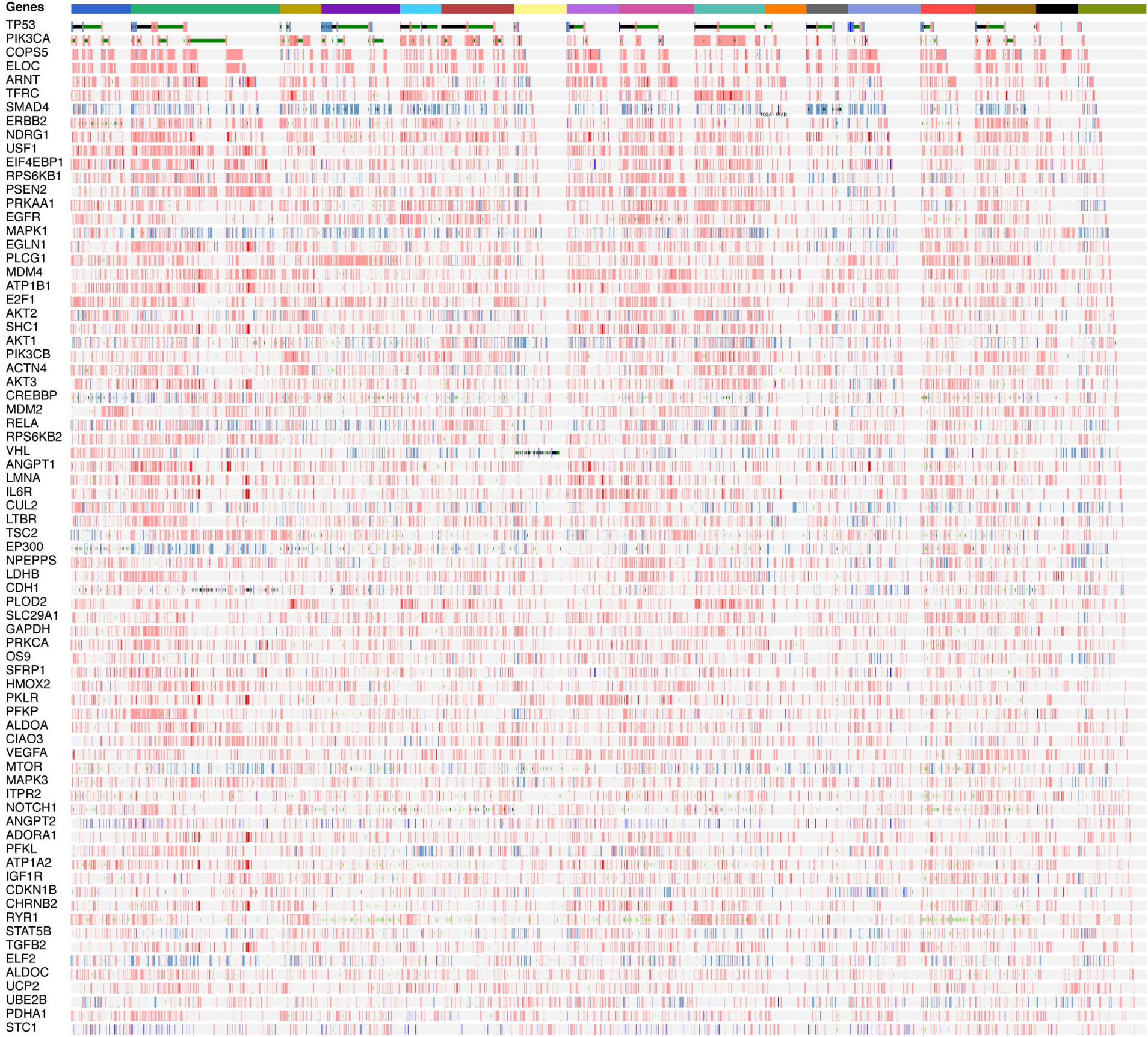

- Inframe mutation (putative driver) - Truncating mutation (putative driver) " Inframe mutation (unknown significance) " Missense mutation (putative driver)

No alterations

- Missense mutation (unknown significance) I Fusion gene | CNV: amplification | CNV: deep deletion m mRNA high [ mRNA low

B

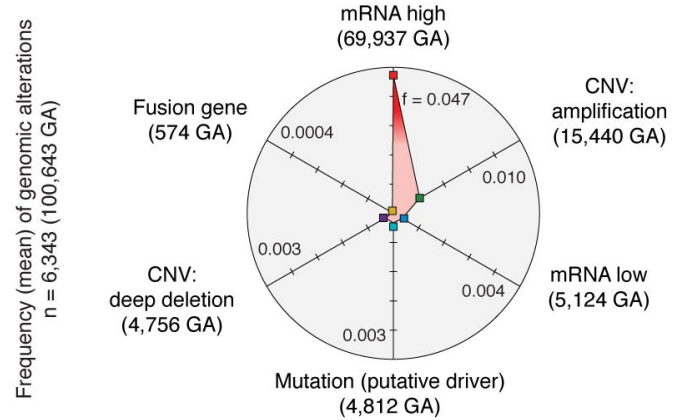

C

mRNA high

mRNA low CNV: amplification

CNV: deep deletion

Mutation

Fusion gene
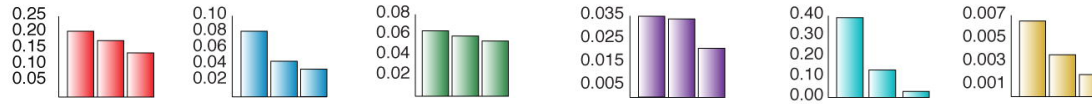

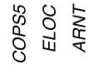

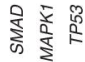

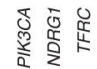

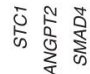

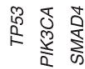




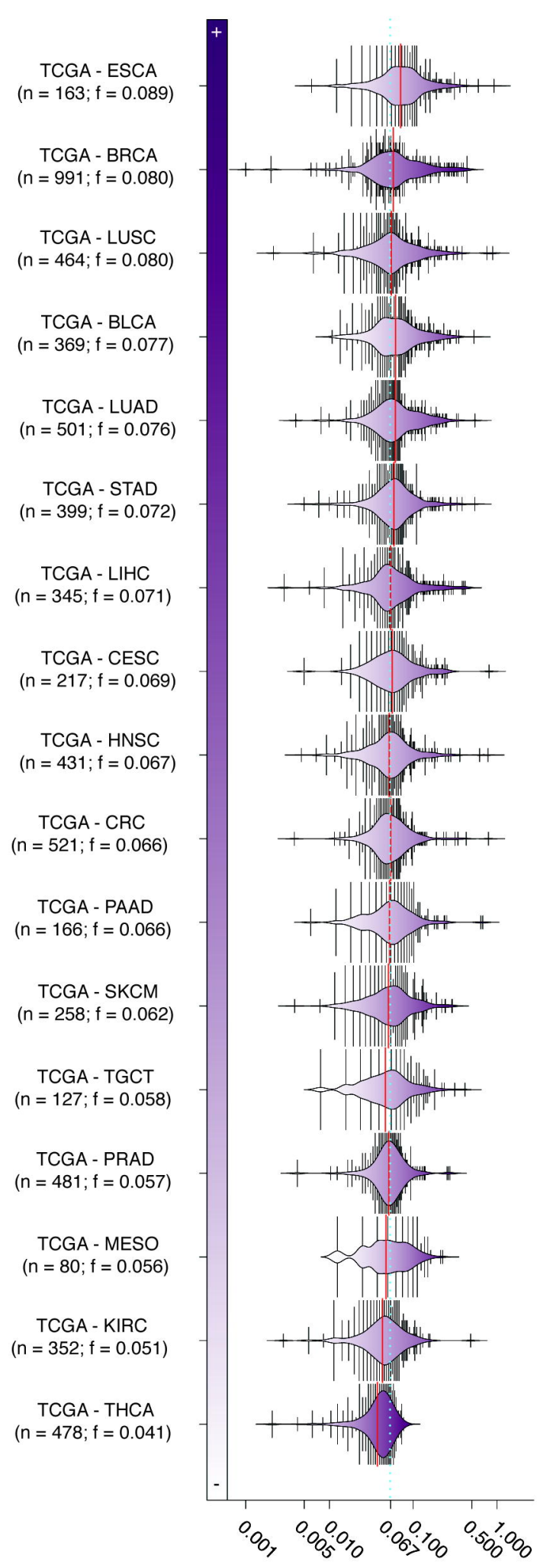

Frequency (mean) of genomic alterations per TCGA - Pan-Cancer type

$(n=6,343 ; f=0.067)$
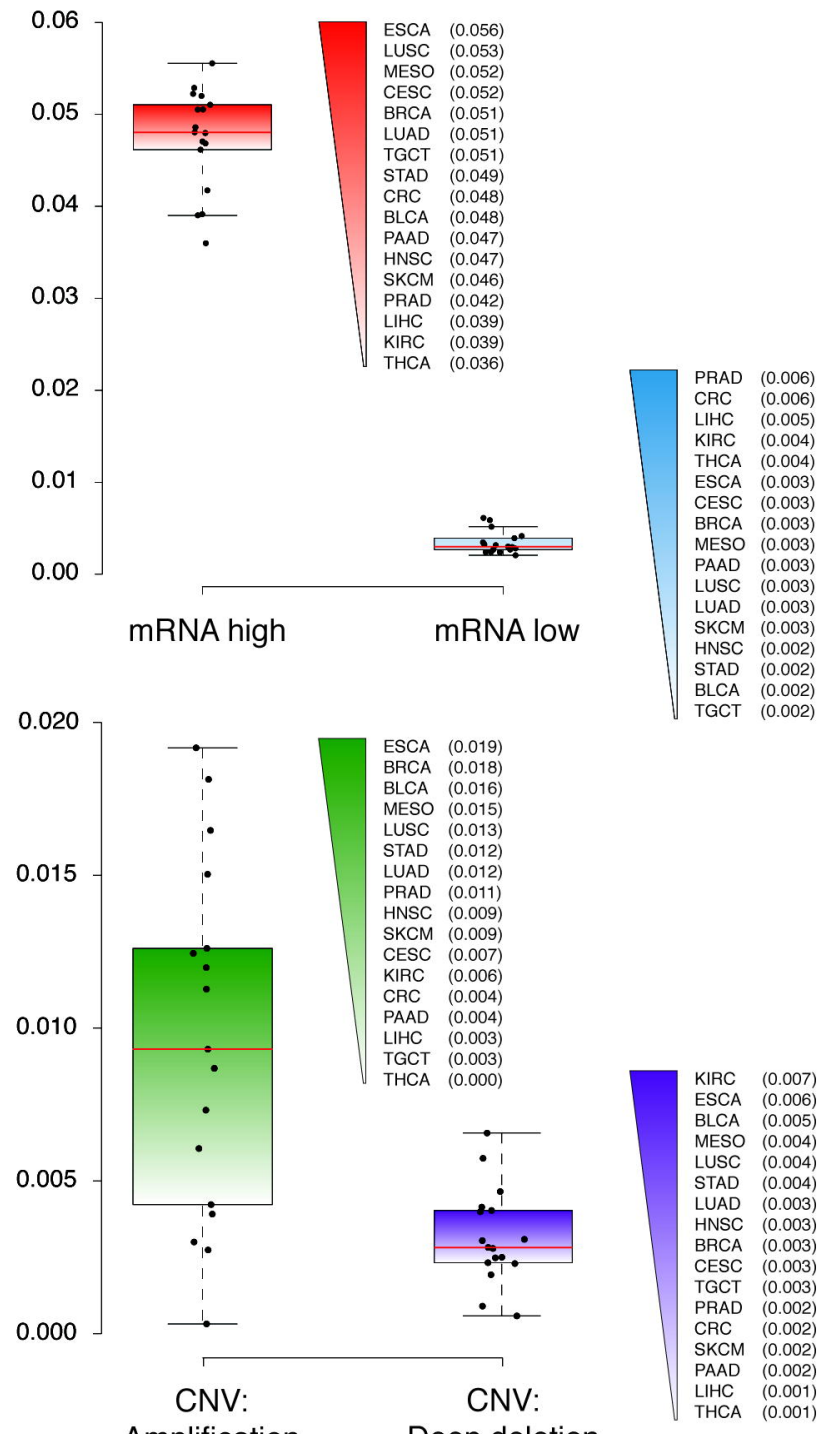


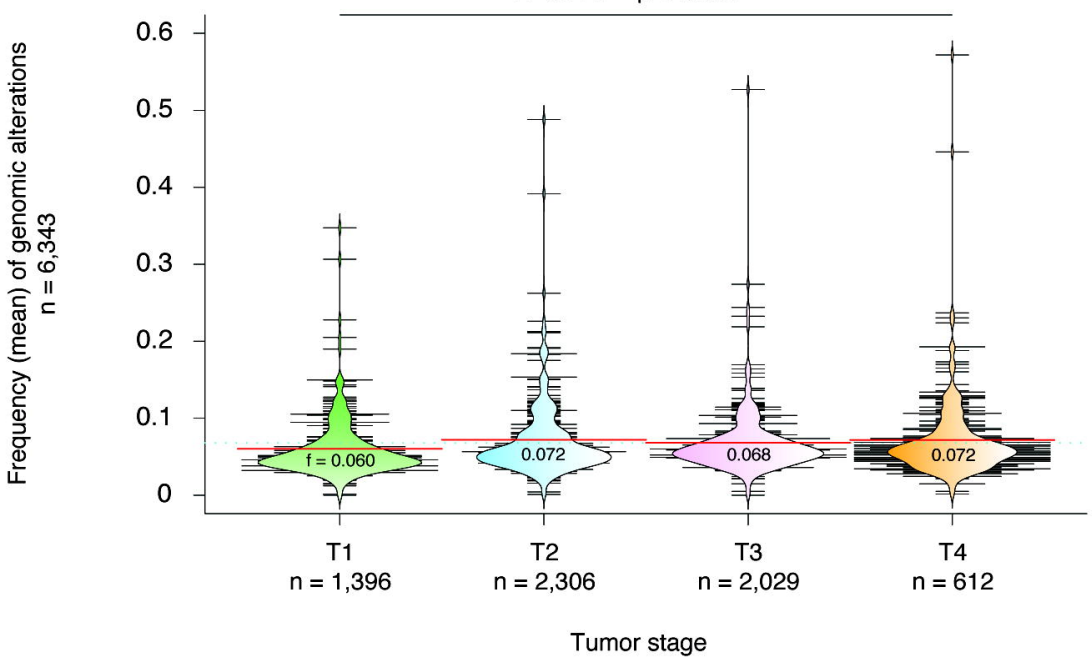

B

CNV: Amplification

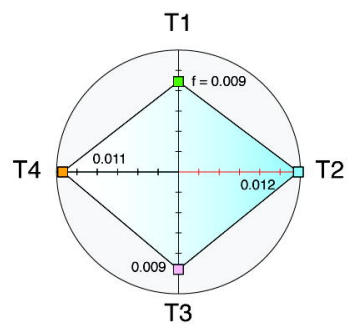

mRNA low

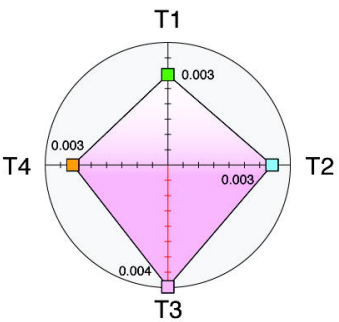

CNV: Deep deletion

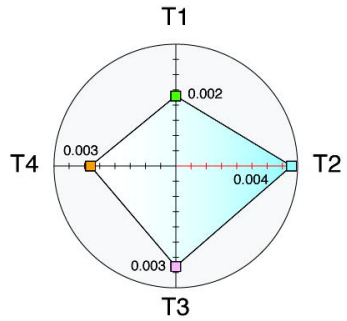

Mutation (putative driver)

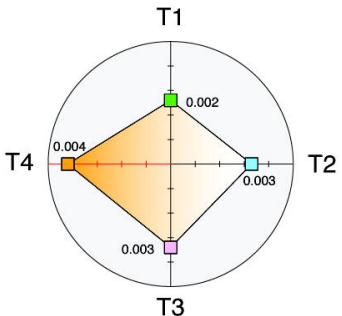

mRNA high

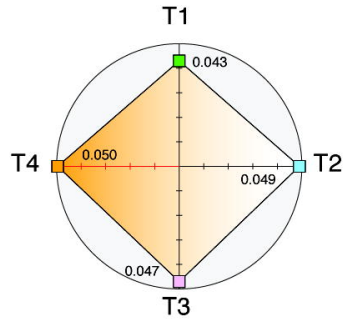

Fusion gene

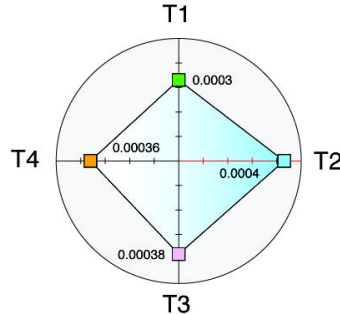

C

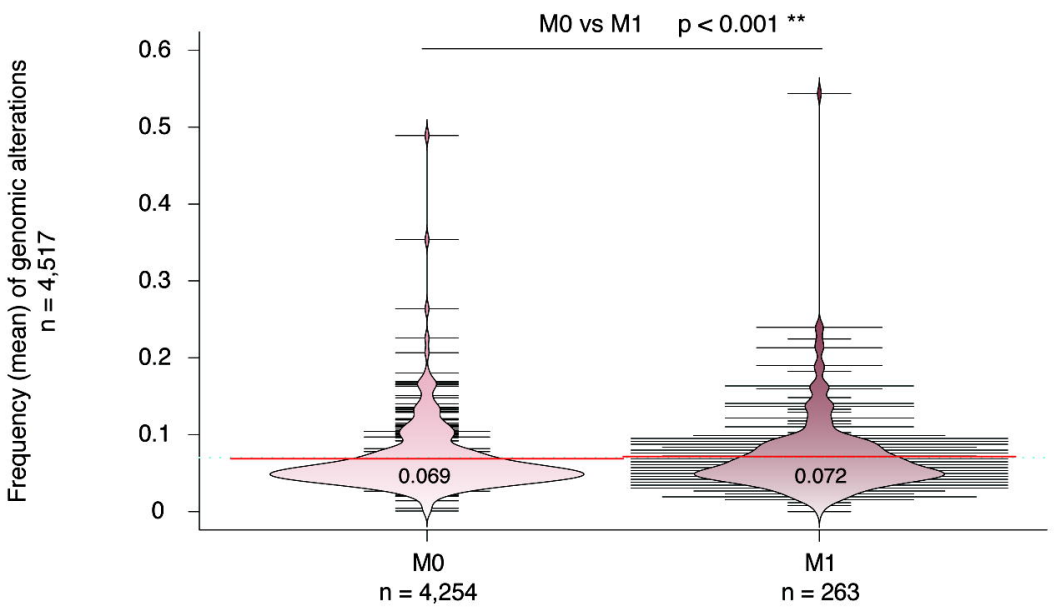

Metastasis status 


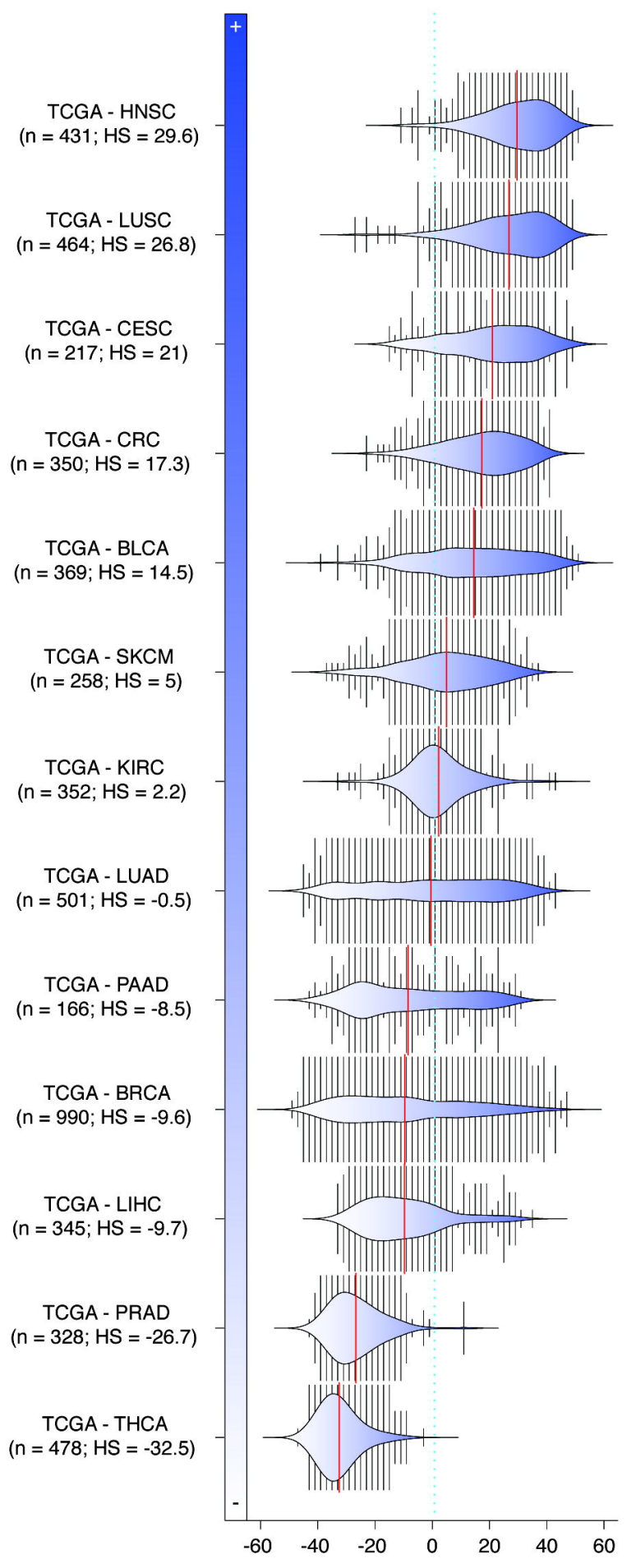

T1 vs T4 $p<0.001$ **

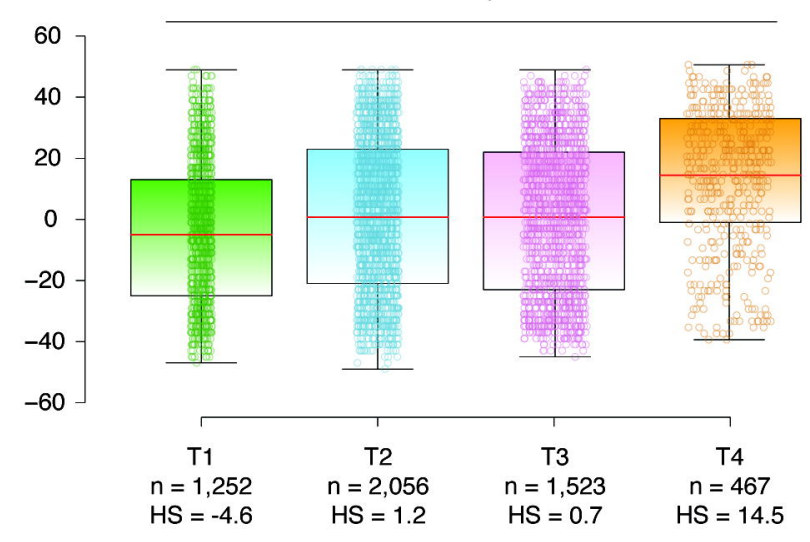

$(n=5,249 ;$ HS mean $=0.85)$
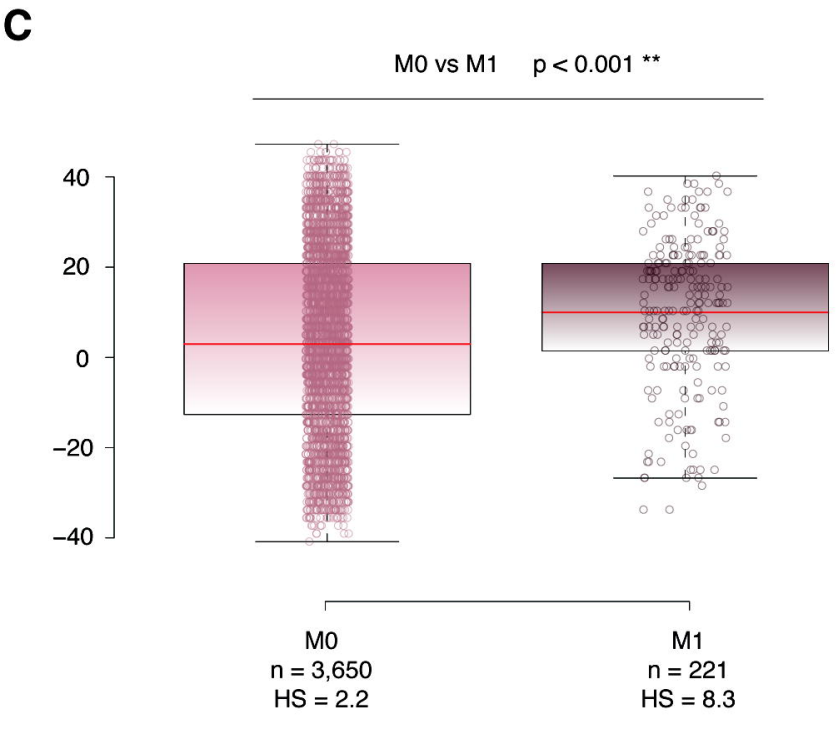

$(n=3,871 ;$ HS mean $=2.5)$

D

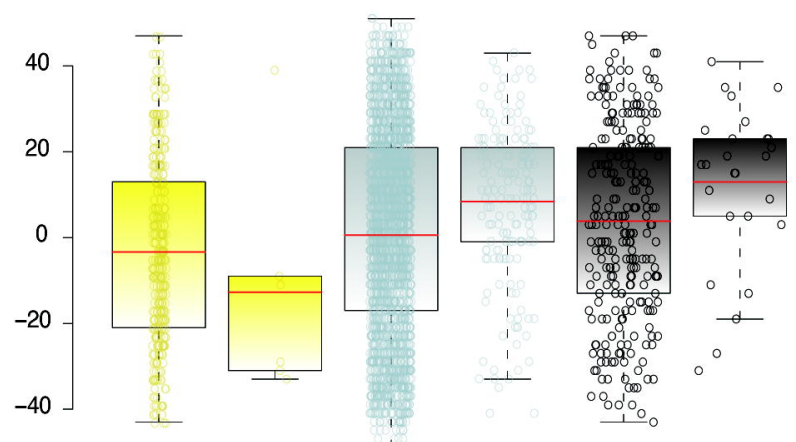

Hypoxia score per TCGA Pan-Cancer type $(n=5,249 ; \mathrm{HS}$ mean $=0.85)$

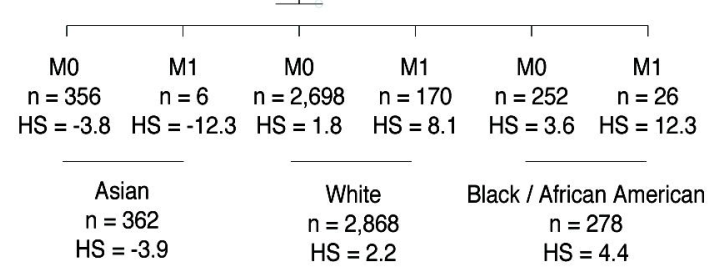




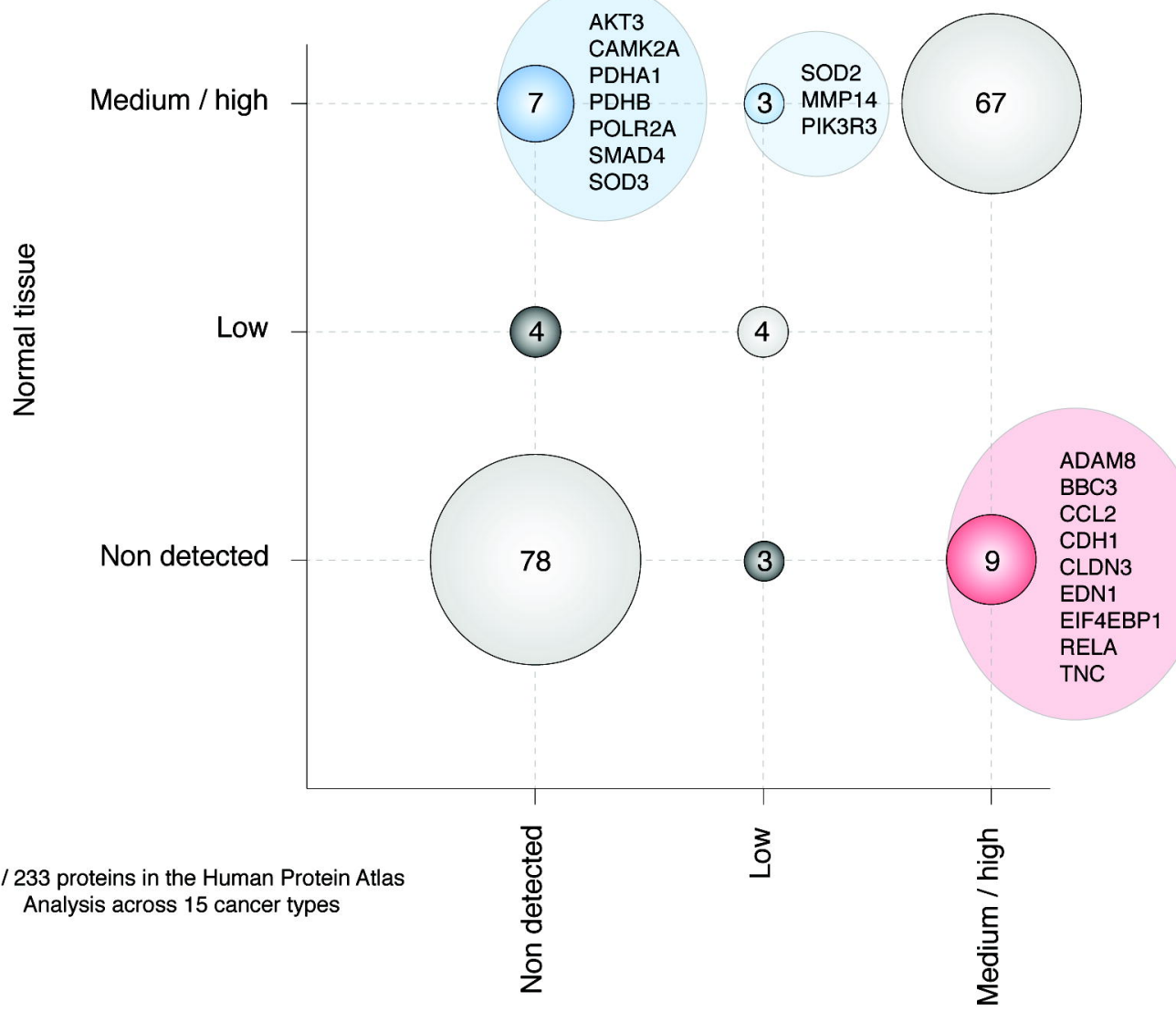

Tumor tissue 


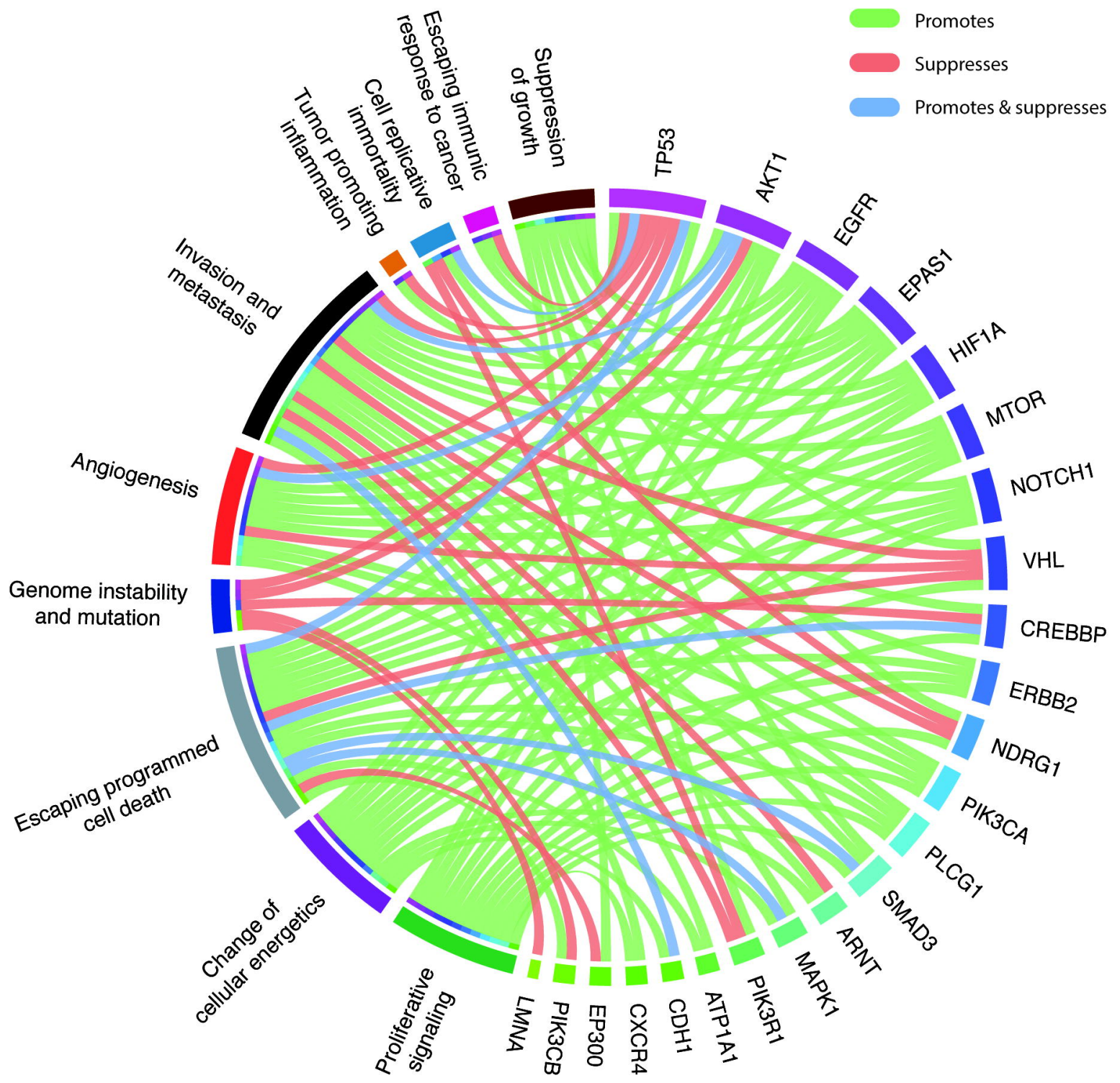




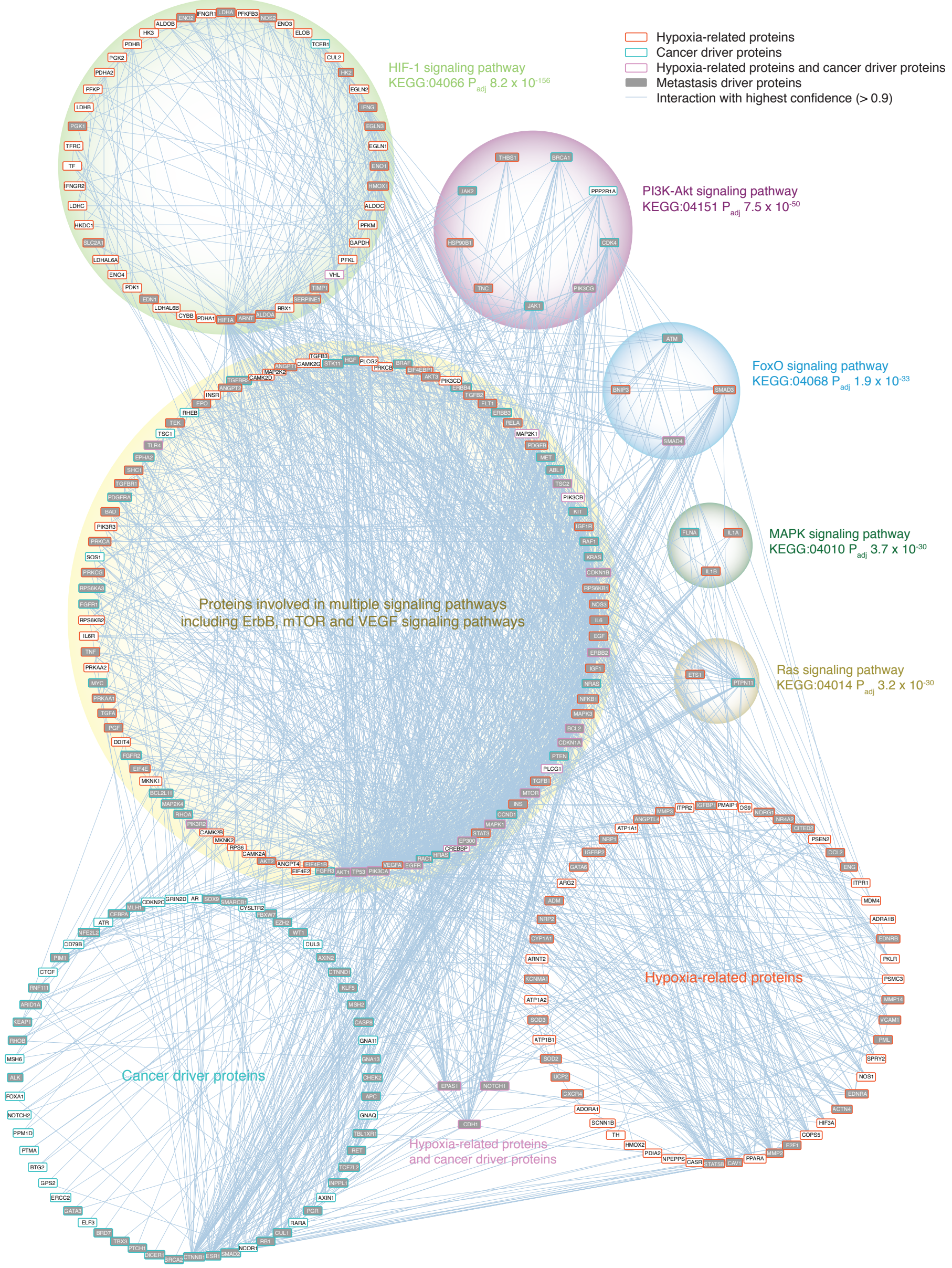




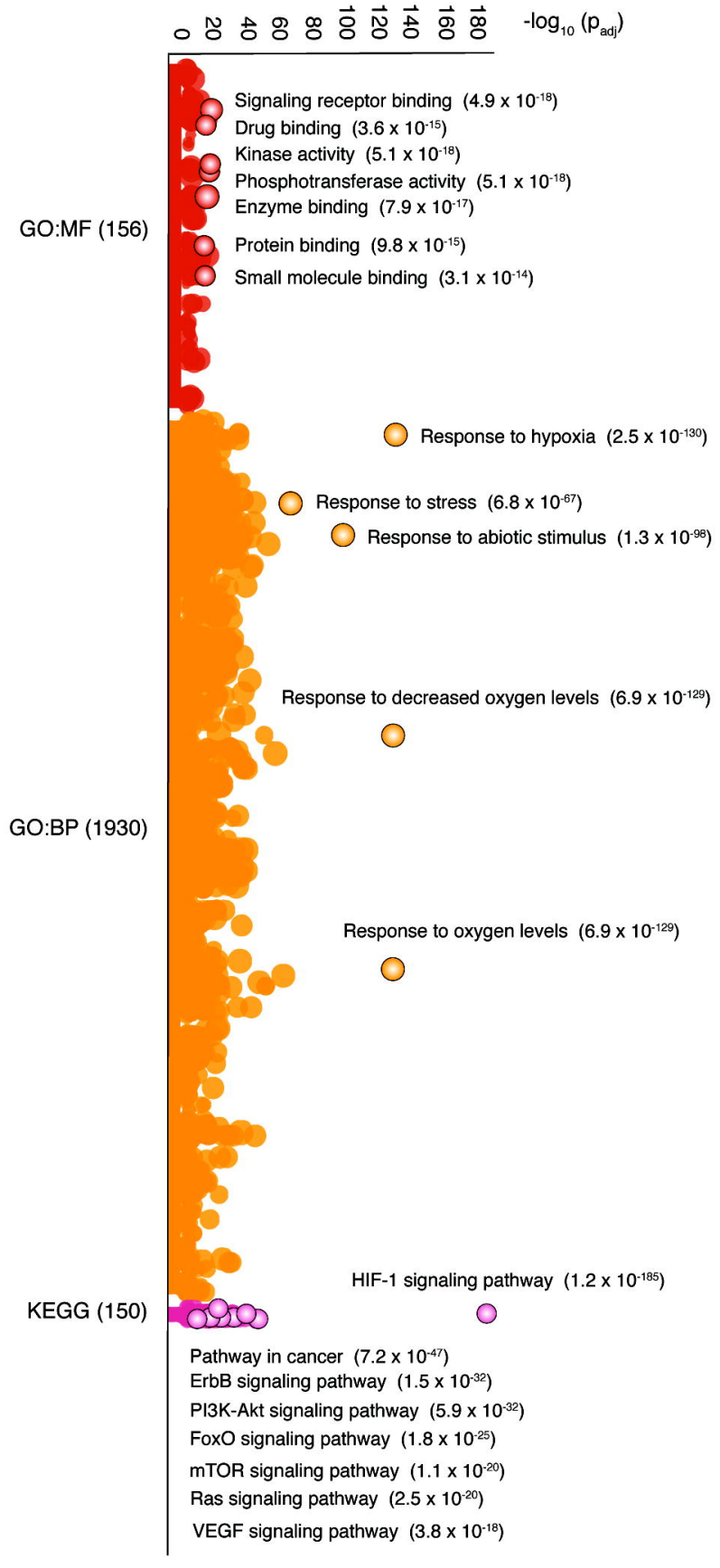

$\mathrm{g}:$ Profiler of 233 hypoxia-related genes

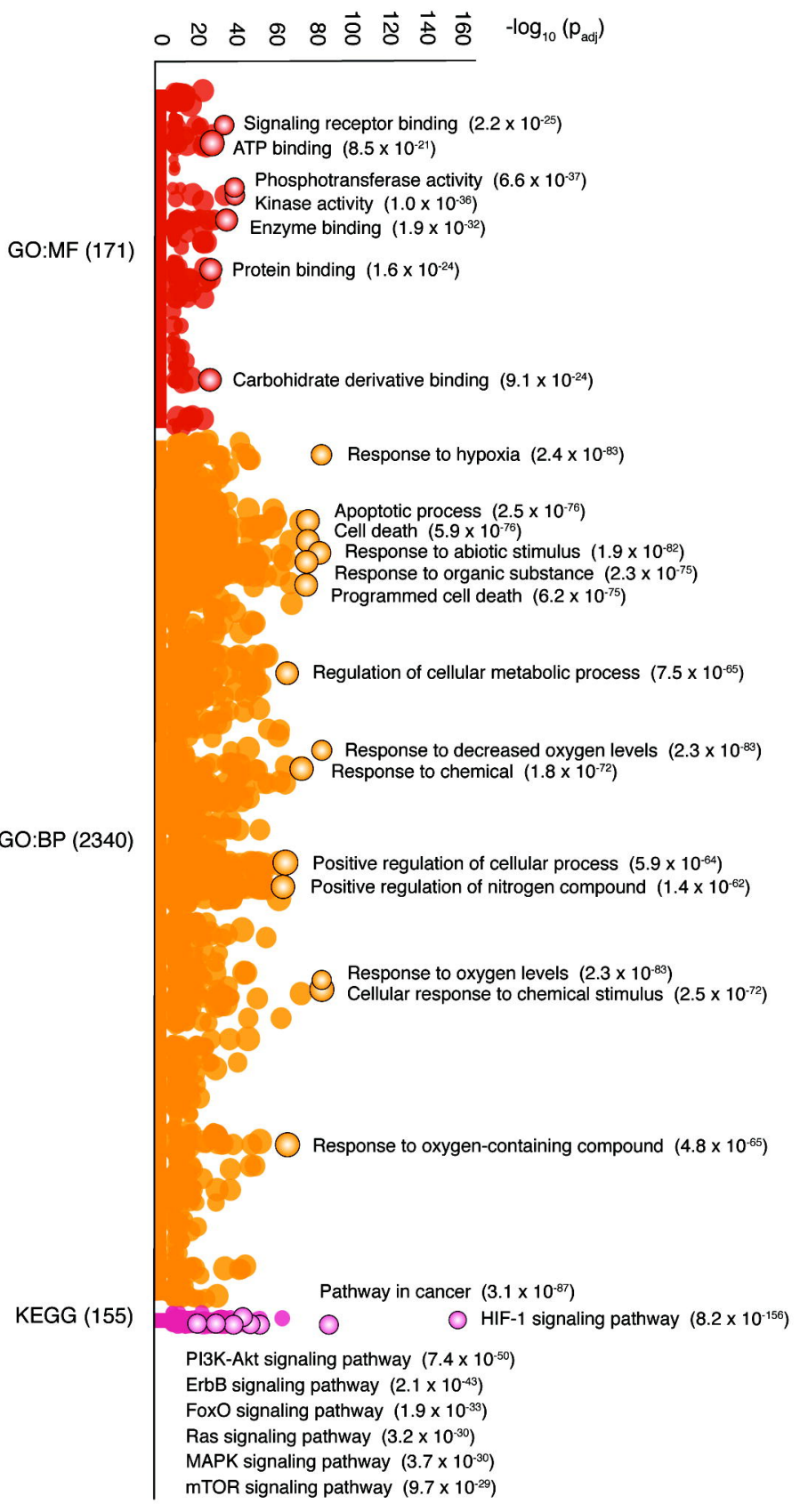

g:Profiler of 285 hypoxia-related genes and cancer driver genes 


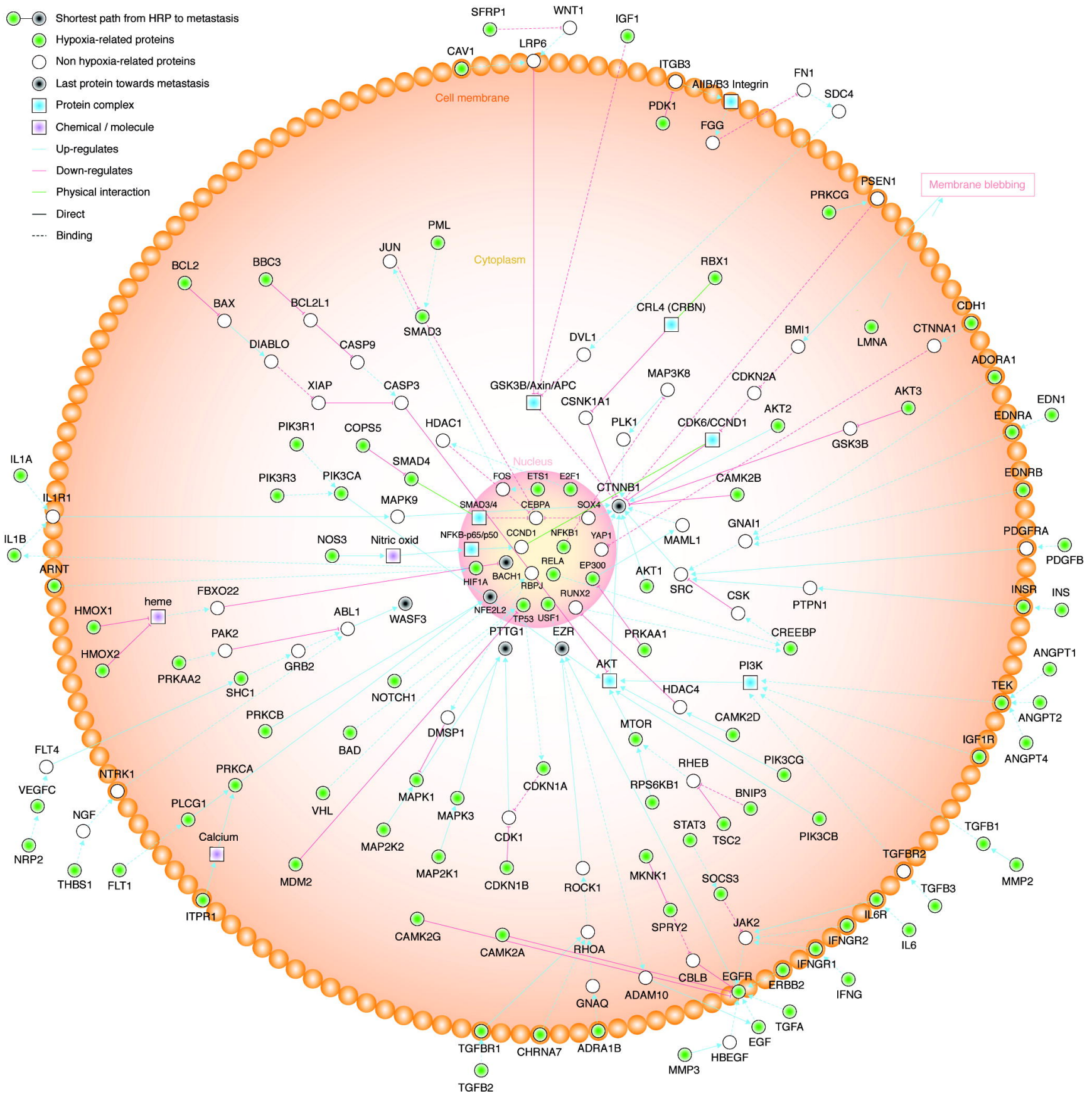




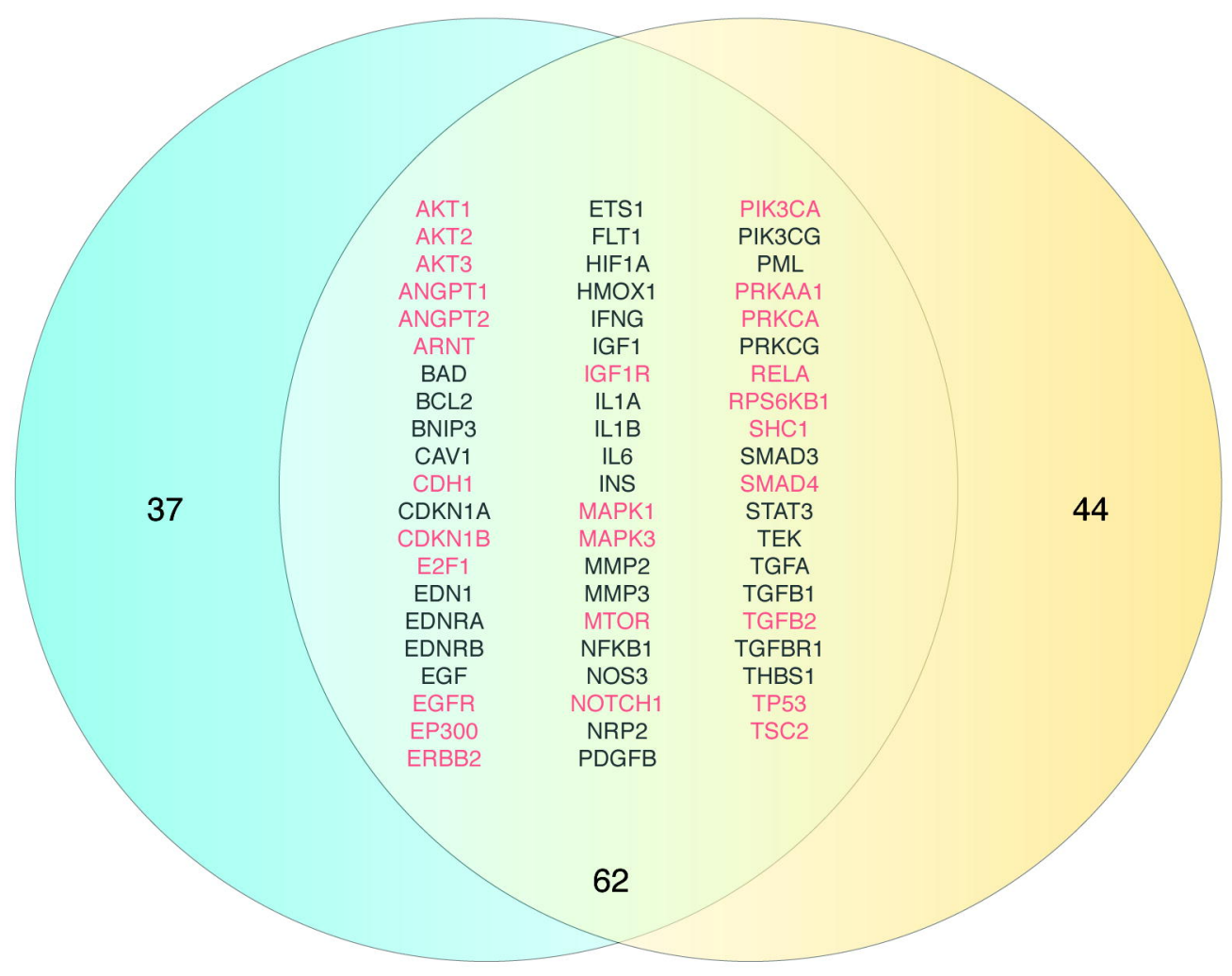

Hypoxia-related proteins whose genes had a frequency mean of genomic alterations more than the average $(>0.068)$ across 17 TCGA Pan-Cancer types 
A

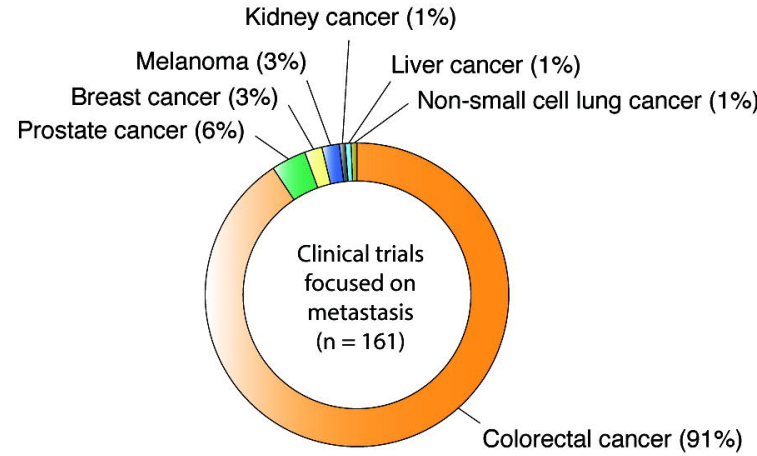

C

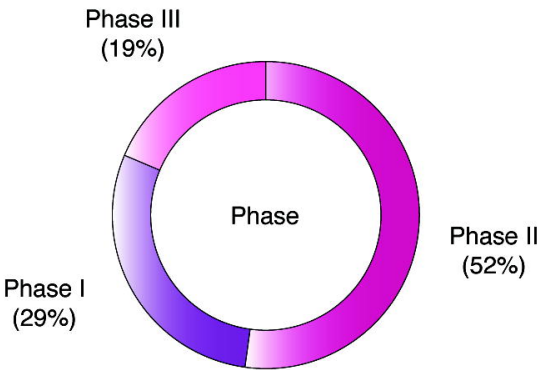

D

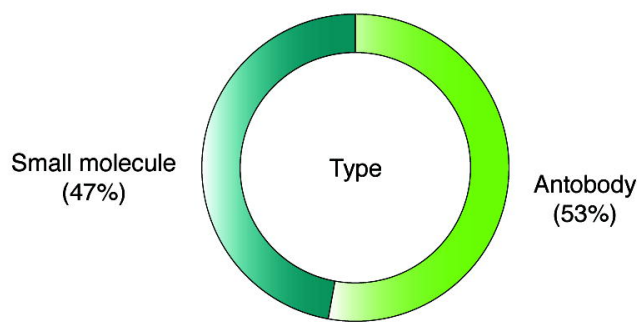

E

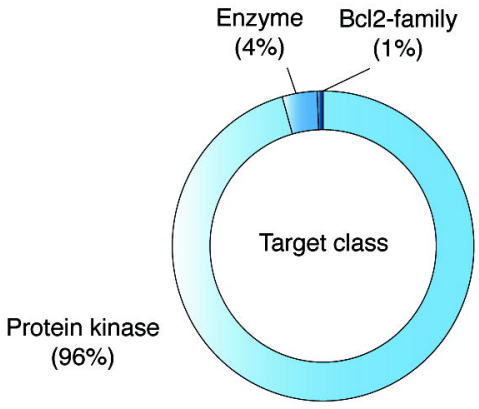

B

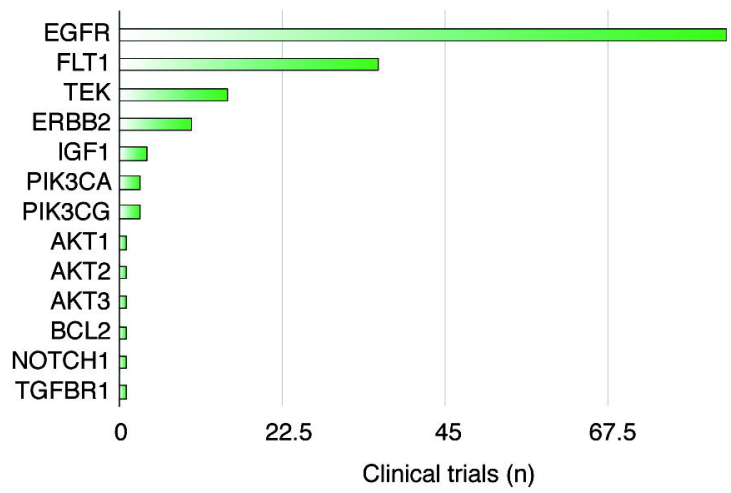

90
$\mathbf{F}$

Clinical trials focused on metastatic disease
Metastatic lung cancer: 1

Metastatic breast cancer: 3 Metastatic kidney cancer: 1

Metastatic liver cancer: 1

Metastatic colorectal cancer: 146

Metastatic melanoma: 3

Metastatic prostate cancer: 6
Dacomitinib: 1

Ly-3023414: 4

Brivanib: 4

Trastuzumab: 3

Panitumumab: 26

Cetuximab: 48

Erlotinib: 2

Lapatinib: 4

Neratinib: 2

Futuximab: 2

Afatinib: 2

Necitumumab: 1

Trastuzumab emitansine: 1

Pertuzumab: 1

Regorafenib: 30

Cediranib: 2

Famitinib: 2

Sorafenib: 5

Tivozanib: 1

Vatalanib: 1

Ganitumab: 2

Dalotuzumab: 1

Linsitinib: 1

Brontictuzumab: 1

Buparlisib: 2

Galunisertib: 1

Sunitinib: 5

Lenvatinib: 1

Ipatasertib: 3

Venetoclax: 1

Gefitinib: 1 